\title{
On the time value of Parisian ruin in (dual) renewal risk processes with exponential jumps
}

\author{
Jeff T.Y. Wong* and Eric C.K. Cheung ${ }^{\dagger}$
}

August 9, 2015

\begin{abstract}
This paper studies the Parisian ruin problem first proposed by Dassios and Wu (2008a,b), where the Parisian ruin time is defined to be the first time when the surplus process has stayed below zero continuously for a pre-specified time length $d$. Both the insurance risk process and the dual model will be considered under exponential distributional assumption on the jump sizes while keeping the inter-arrival times arbitrary. In these two models, the Laplace transform of the Parisian ruin time is derived by extending the excursion techniques in Dassios and Wu (2008a) and taking advantage of the memoryless property exponential distributions. Our results are represented in integral forms, which are expressed in terms of the (joint) densities of various ruin-related quantities that are available in the literature or obtainable using the Lagrange's expansion theorem. As a by-product, we also provide the joint distribution of the numbers of periods of negative surplus that are of duration more than $d$ and less than $d$, which can be obtained using some of our intermediate results. The case where the Parisian delay period $d$ is replaced by a random time is also discussed, and it is applied to find the Laplace transform of the occupation time when the surplus is negative. Numerical illustrations concerning an Erlang(2) insurance risk model are given at the end.
\end{abstract}

Keywords: Parisian ruin time; Sparre Andersen model; Dual risk model; Lagrange's expansion theorem; Excursion; Occupation time in red.

\section{Introduction}

In this paper, the surplus process $\{U .(t)\}_{t \geq 0}$ of a business enterprise is generally modelled by

$$
U .(t)=u \pm X(t), \quad t \geq 0,
$$

where $U .(0)=u \geq 0$ is the initial surplus and $X(t)=c t-\sum_{i=1}^{N(t)} Y_{i}$ with $c>0$. It is assumed that $\left\{Y_{i}\right\}_{i=1}^{\infty}$ is a sequence of independent and identically distributed (i.i.d.) positive jumps, and $\{N(t)\}_{t \geq 0}$ is a renewal process that is independent of $\left\{Y_{i}\right\}_{i=1}^{\infty}$ and characterized by the sequence of i.i.d. inter-arrival times $\left\{V_{i}\right\}_{i=1}^{\infty}$. In the rest of the paper, it is assumed that each $Y_{i}$ is exponentially distributed with

\footnotetext{
* Department of Statistics and Actuarial Science, University of Waterloo, 200 University Avenue West, Waterloo, Ontario, Canada, N2L 3G1.

${ }^{\dagger}$ Department of Statistics and Actuarial Science, University of Hong Kong, Pokfulam, Hong Kong. Corresponding author: eckc@hku.hk
} 
mean $1 / \mu$. For later use, we define $k(\cdot)$ to be the density of $V_{i}$ with corresponding Laplace transform $\widetilde{k}(s)=\int_{0}^{\infty} e^{-s t} k(t) \mathrm{d} t$.

Depending on the nature of the business, (1.1) takes on different signs. For a business that receives income at a constant rate over time and faces losses that occur randomly in both time and amount, (1.1) takes a positive sign and we denote

$$
U_{S}(t)=u+X(t)=u+c t-\sum_{i=1}^{N(t)} Y_{i},
$$

where the subscript ' $S$ ' stands for 'standard' model. A typical example of business enterprise with surplus process that falls into this category is an insurance company. In this case, $c>0$ can be interpreted as the incoming premium rate per unit time, $Y_{i}$ represents the amount of the $i$-th insurance claim from the policyholders, and $N(t)$ is the number of insurance claims until time $t$. Surplus process with model dynamics described by (1.2) is commonly referred to as the Sparre Andersen risk model (Sparre Andersen (1957)) or renewal model. If $\{N(t)\}_{t \geq 0}$ is a Poisson process, then $\left\{U_{S}(t)\right\}_{t \geq 0}$ reduces to the classical compound Poisson risk process. The time of ruin of the process $\left\{U_{S}(t)\right\}_{t \geq 0}$ is typically defined to be $T_{U_{S}}=\inf \left\{t>0: U_{S}(t)<0\right\}$ with the convention that $\inf \{\emptyset\}=\infty$. The analysis of the ruin probability, the Gerber-Shiu expected discounted penalty function (Gerber and Shiu (1998)) and other ruin-related quantities in Sparre Andersen models can be found in e.g. Dickson and Hipp (2001), Li and Garrido (2004), Gerber and Shiu (2005), Albrecher and Teugels (2006), Willmot (2007), Asmussen and Albrecher (2010, Chapter IX.4), Cheung et al. (2010), Willmot and Woo (2010), and Cheung (2013).

In contrast, the case of a negative sign in (1.1) is suitable for businesses with a constant rate of expense $c$ and gains occurring randomly in both time and amount. The surplus process is thus given by

$$
U_{D}(t)=u-X(t)=u-c t+\sum_{i=1}^{N(t)} Y_{i}
$$

where the subscript ' $D$ ' stands for 'dual' risk model (as a reflection of the standard model). As commented by e.g. Avanzi et al. (2007), the dual model (1.3) is appropriate for pharmaceutical and petroleum companies, and each upward jump can be interpreted as the net present value of future income resulting from a discovery or an invention. The ruin time of $\left\{U_{D}(t)\right\}_{t \geq 0}$ is given by $T_{U_{D}}=\inf \left\{t>0: U_{D}(t)=0\right\}$. While classical ruin probability results are available in e.g. Cramér (1955, Section 5.13), Takács (1967, pp. 152-154), Seal (1969, pp. 116-119) when $\{N(t)\}_{t \geq 0}$ follows a Poisson process, the dual Sparre Andersen risk model was studied by e.g. Mazza and Rullière (2004), Albrecher et al. (2008, Appendix), and Cheung (2012).

Traditionally, the analyses of the risk processes in the literature have been mostly conducted under the assumption that ruin occurs immediately once the surplus drops below zero. However, in practice the ruin probability is usually very small, and even if ruin occurs the company can usually continue its business and survive negative surplus for some time in the hope of a quick recovery. See comments in e.g. Gerber (1990), Egidio dos Reis (1993) and Cheung (2012). Recently, there have been increased research interests in risk models (particularly in the insurance risk process (1.2)) with modified definitions of ruin that possibly allow the company's surplus to stay negative without declaring ruin. One of these earliest concepts is 'absolute ruin' (Dassios and Embrechts (1989)), for which the company can borrow money at a certain debit interest as long as the drift of the process is positive (and absolute ruin occurs when the 
drift becomes negative). See also e.g. Cai (2007), Gerber and Yang (2007), Cai et al. (2009, Section 7), and Cheung (2011, Section 6). Another development is the idea of randomized observations proposed by Albrecher et al. (2011a, 2013), where the event of ruin is only monitored at $\operatorname{Erlang}(n)$ intervals as the company balances its books on a periodic basis. See also Albrecher and Ivanovs (2013) and Albrecher et al. (2014) for further results concerning a Poissonian observer. A model related to randomized observations is the (Gamma-)Omega model in Albrecher et al. (2011b), Gerber et al. (2012), and Albrecher and Lautscham (2013), who assumed that the company declares bankruptcy with probability $\omega(x) \mathrm{d} t$ if the surplus level is $x<0$ at time $(t, t+\mathrm{d} t]$, where $\omega(\cdot)$ is the bankruptcy rate function (see Section 5 ).

In this paper, we focus on the concept of Parisian ruin proposed by Dassios and Wu (2008a,b) in the context of a compound Poisson insurance risk model. According to their definition, the Parisian ruin time is defined as the first time when the surplus process has stayed below zero continuously for a prescribed length of time $d>0$. Their idea was motivated by the Parisian options in finance (see e.g. Chesney et al. (1997) and Schröder (2003)). While Dassios and Wu (2008a) derived the exact Laplace transform of the Parisian ruin time under exponential claims and provided a diffusion approximation as well, Dassios and $\mathrm{Wu}$ (2008b) showed that a Cramér-Lundberg type asymptotic formula also holds true for the Parisian ruin probability when claims are light-tailed. Their results were extended to general spectrally negative Lévy insurance risk processes by Czarna and Palmowski (2011) and Loeffen et al. (2013). When the constant $d$ is instead replaced by (a sequence of) mixed Erlang random variables, Landriault et al. (2014) studied the Laplace transform of the Parisian ruin time for a Lévy insurance risk model with bounded variation. Another extension was also made by Czarna (2014) in the Lévy model, where ruin is declared once the surplus process stays negative continuously for a period of $d$ or goes below a fixed negative level - a. Note that Parisian ruin problems have so far only been analyzed in continuous-time risk models in the literature, with the exception of Czarna et al. (2014) who looked at the compound binomial model.

This paper is organized as follows. In Section 2, the Parisian ruin time and notations in relation to excursions are formally defined. An auxiliary process resembling the dual model (1.3) will also be defined as in Cheung (2012), and some discounted/joint densities of various ruin-related quantities required later are introduced as well. These densities are either available in the literature or obtainable via the Lagrange's expansion theorem. This lays the foundation for a systematic analysis of the Parisian ruin problems in subsequent sections. In Section 3, the main results regarding the Laplace transform of the Parisian ruin time are presented. Two models will be considered: the standard insurance model (1.2) and the dual model (1.3) when the inter-arrival times are kept general and the jump sizes are exponentially distributed (as opposed to Lévy models in the literature). Our derivation is based on an extension of the excursion techniques used in Dassios and Wu (2008a), and the memoryless property of exponential distributions plays an important role. We shall demonstrate that the ideas are applicable by appropriately partitioning the sample path of the surplus process into segments that are mutually independent. The results are generally represented in integral forms in terms of the densities given in Section 2. Section 4 is concerned with a joint distribution in relation to the numbers of periods of negative surplus (that are of duration more than $d$ and less than $d$ ), which follows as a by-product using our intermediate findings. Section 5 utilizes the results in Section 3 to state the Laplace transform of the Parisian ruin time when the deterministic delays are replaced by a sequence of i.i.d. random times. In particular, under exponential delays, this is applied to derive a neat expression for the Laplace transform of the occupation time when the surplus is negative (i.e. the time in red). In Section 6, we provide an example concerning the Erlang(2) insurance risk model with exponential claims, and show how the integrals involved in the Laplace transform of the Parisian ruin time can be conveniently computed. Numerical illustrations will be given. Section 7 concludes the paper with some open research problems. 


\section{Preliminaries}

\subsection{Parisian ruin and excursions}

Following Dassios and Wu (2008a), we start by defining various stopping times and introducing the idea of excursions that are applicable to the general model (1.1). First, the time of ruin is given by $\tau_{U}^{-, 1}=\inf \left\{t>0: U_{.}(t)<0\right\}$ whereas the first recovery time is defined by $\tau_{U}^{+, 1}=\inf \left\{t>\tau_{U}^{-, 1}: U .(t)>0\right\}$. For $n=2,3, \ldots$, we further define $\tau_{U}^{-, n}=\inf \left\{t>\tau_{U}^{+, n-1}: U .(t)<0\right\}$ to be the $n$-th time the surplus drops below zero and $\tau_{U}^{+, n}=\inf \left\{t>\tau_{U}^{-, n}: U_{.}(t)>0\right\}$ to be the $n$-th recovery time. Also let $\tau_{U .}^{-, 0}=\tau_{U}^{+, 0}=0$. Then, for $j=1,2, \ldots$, the time interval between $\tau_{U}^{+, j-1}$ and $\tau_{U}^{-, j}$ constitutes the $j$-th excursion of $\{U .(t)\}_{t \geq 0}$ above zero whereas the interval between $\tau_{U}^{-, j}$ and $\tau_{U}^{+, j}$ is the $j$-th excursion below zero. Letting $L=\inf \left\{n \in \mathbb{N}: \tau_{U}^{+, n}-\tau_{U}^{-, n} \geq d\right\}$ (where $\mathbb{N}$ is the set of natural numbers), the Parisian ruin time $\tau_{U}$. can be defined as

$$
\tau_{U .}= \begin{cases}\tau_{U .}^{-, L}+d=\sum_{j=1}^{L}\left(\tau_{U .}^{-, j}-\tau_{U .}^{-, j-1}\right)+d, & L<\infty . \\ \infty, & L=\infty .\end{cases}
$$

Clearly, if $L<\infty$ then $L$ represents the number of excursions above zero before Parisian ruin whereas $L=\infty$ (i.e. $\tau_{U} .=\infty$ ) means that Parisian ruin does not occur. Sample paths for the insurance risk process $\left\{U_{S}(t)\right\}_{t \geq 0}$ and the dual model $\left\{U_{D}(t)\right\}_{t \geq 0}$ are depicted in Figures 1 and 2 respectively.

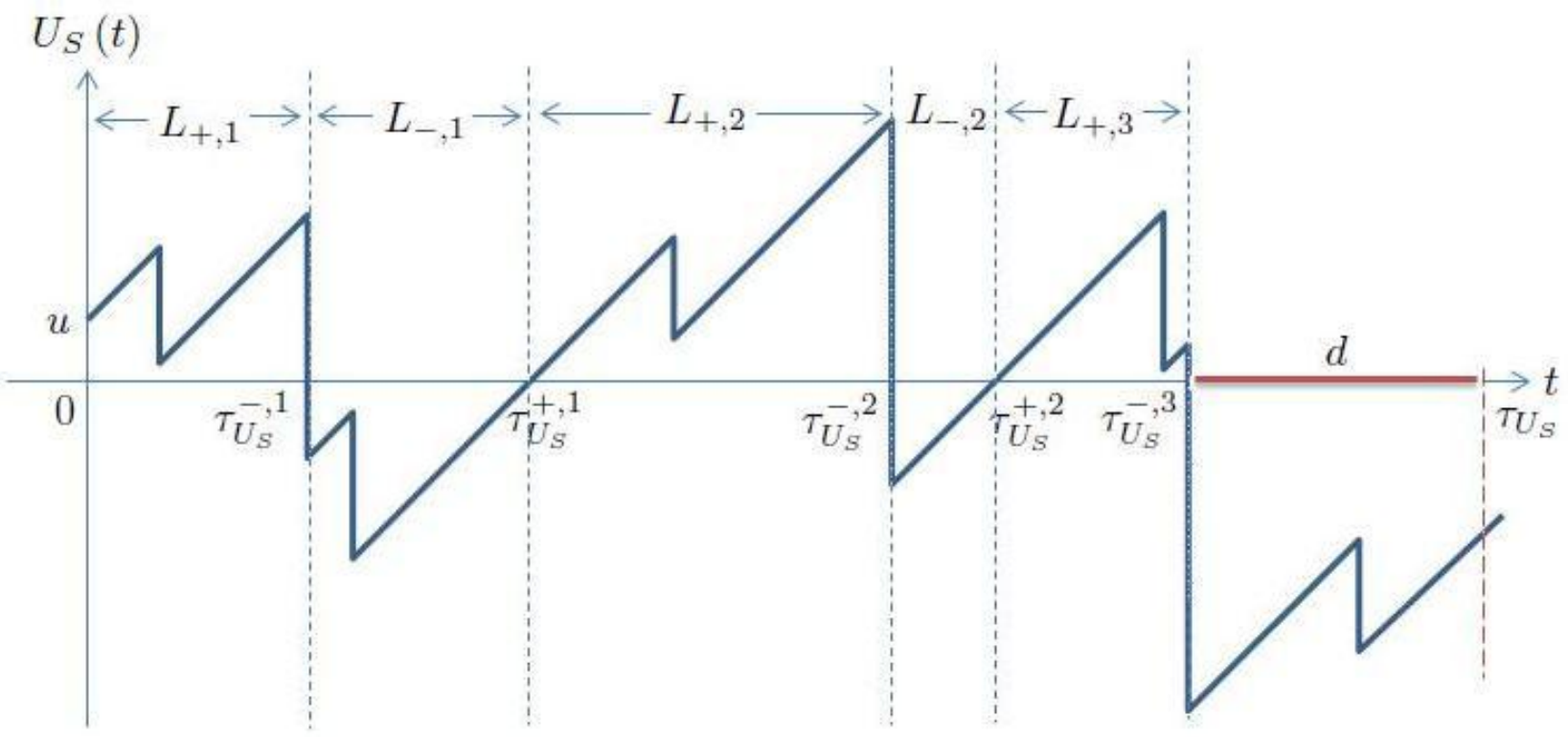

Figure 1: Sample path of $\left\{U_{S}(t)\right\}_{t \geq 0}$

The key quantity of interest in this paper is the Laplace transform of the Parisian ruin time given by

$$
\psi_{\delta, U .}(u)=\mathbb{E}^{u}\left[e^{-\delta \tau_{U}} 1_{\left\{\tau_{U}<\infty\right\}}\right], \quad u \geq 0,
$$

where $\delta \geq 0$ is the Laplace transform argument, $1_{A}$ is the indicator function of the event $A$, and $\mathbb{E}^{u}$ represents the expectation taken under the initial condition $U .(0)=u$. Clearly, $\psi_{\delta, U}$. $(u)$ reduces to the (ultimate) Parisian ruin probability when $\delta=0$. To ease our upcoming analysis, for $j=1,2, \ldots$ we define the following random times pertaining to the process $\{U .(t)\}_{t \geq 0}$, namely

$$
L_{+, j}=\tau_{U}^{-, j}-\tau_{U}^{+, j-1}=\text { Length of the } j \text {-th excursion above zero, }
$$




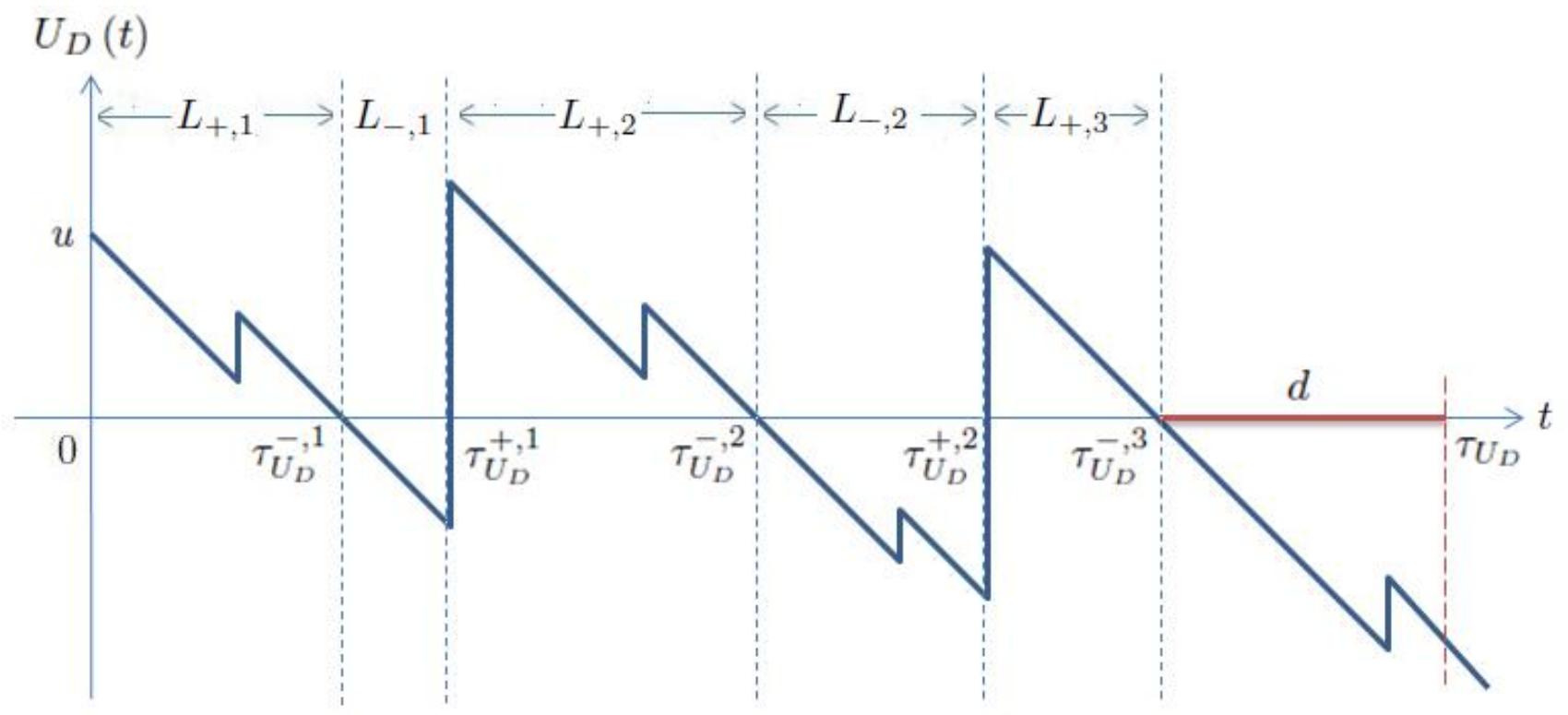

Figure 2: Sample path of $\left\{U_{D}(t)\right\}_{t \geq 0}$

$L_{-, j}=\tau_{U}^{+, j}-\tau_{U}^{-, j}=$ Length of the $j$-th excursion below zero,

as well as the events

$$
\begin{aligned}
& B_{j}=\left\{L_{+, j}<\infty\right\}=\text { Event that the } j \text {-th excursion above zero is of finite time length. } \\
& C_{j}=\left\{L_{-, j}<d\right\}=\text { Event that the } j \text {-th excursion below zero is of length less than } d .
\end{aligned}
$$

Hence, when Parisian ruin occurs, the Parisian ruin time $\tau_{U}$. defined by (2.1) admits the representation

$$
\tau_{U .}=\sum_{j=1}^{i} L_{+, j}+\sum_{j=1}^{i-1} L_{-, j}+d \text { on the set } A_{i},
$$

where the set $A_{i}$ is given by

$A_{i}=$ Event that the first time the length of excursion below zero reaches $d$ happens in the $i$-th excursion

$$
= \begin{cases}B_{1} \cap C_{1}^{c} & i=1 . \\ \left(\bigcap_{j=1}^{i} B_{j}\right) \cap\left(\bigcap_{j=1}^{i-1} C_{j}\right) \cap C_{i}^{c}, & i=2,3, \ldots\end{cases}
$$

Because the set $\left\{\tau_{U},<\right\}$ is equivalent to $\bigcup_{i=1}^{\infty} A_{i},(2.2)$ can be rewritten as

$$
\psi_{\delta, U .}(u)=\sum_{i=1}^{\infty} \mathbb{E}^{u}\left[e^{-\delta \tau_{U}} \cdot 1_{A_{i}}\right]
$$

In other words, to determine the Laplace transform of the Parisian ruin time, it is sufficient to evaluate $\mathbb{E}^{u}\left[e^{-\delta \tau_{U}} \cdot 1_{A_{i}}\right]$. This will be performed in the Section 3 for the (dual) Sparre Andersen model with exponential jumps. We remark that the discussion in this subsection is also applicable to the (dual) compound Poisson model with general jump distribution, where the determination of $\mathbb{E}^{u}\left[e^{\left.-\delta \tau_{U} \cdot 1_{A_{i}}\right] \text { can }}\right.$ be found in Wong (2014, Chapters 2.5.3 and 2.5.4). 


\subsection{Other ruin-related random variables and an auxiliary process}

To analyze renewal models in Section 3 where the density $k(\cdot)$ of the inter-arrival times $\left\{V_{i}\right\}_{i=1}^{\infty}$ is kept general, apart from the idea of excursions we need to rely on some new random variables and an auxiliary process introduced in Cheung (2012). For convenience, we shall follow closely the notations therein. First, for the dual model $\left\{U_{D}(t)\right\}_{t \geq 0}$ in (1.3), the random time $T_{U_{D}}^{*}=\sum_{i=1}^{N\left(T_{U_{D}}\right)+1} V_{i}$ represents the time of the first gain after the (traditional) ruin time and $\left|U_{D}\left(T_{U_{D}}^{*-}\right)\right|$ is the (absolute) amount of the shortfall immediately before $T_{U_{D}}^{*}$. Note that the ruin time is related to these variables via $T_{U_{D}}=$ $T_{U_{D}}^{*}-\left|U_{D}\left(T_{U_{D}}^{*-}\right)\right| / c$ (see Cheung (2012, Figure 1)). The joint distribution of $\left(T_{U_{D}}^{*},\left|U_{D}\left(T_{U_{D}}^{*-}\right)\right|\right)$ given $U_{D}(0)=u \geq 0$ consists of two parts. Specifically, for ruin occurring without any upward jump, one has that $T_{U_{D}}^{*}=\left(u+\left|U_{D}\left(T_{U_{D}}^{*-}\right)\right|\right) / c$. In this case, the marginal density of $\left|U_{D}\left(T_{U_{D}}^{*-}\right)\right|$ at $y>0$ is sufficient to characterize the joint distribution, and it is given by $(1 / c) k((u+y) / c)$. In contrast, if there is at least one jump before ruin, then there is no simple relationship between $T_{U_{D}}^{*}$ and $\left|U_{D}\left(T_{U_{D}}^{*-}\right)\right|$, and the density at $(t, y)$ for $y>0$ and $t>(u+y) / c$ is denoted by $f_{U_{D}}(t, y \mid u)$.

In relation to the process $\left\{U_{D}(t)\right\}_{t \geq 0}$ is the modified dual process $\left\{Z_{D}(t)\right\}_{t \geq 0}$ defined by

$$
Z_{D}(t)=z+Y_{1}-c t+\sum_{i=1}^{M(t)} Y_{i+1}, \quad t \geq 0,
$$

where $Z_{D}\left(0^{-}\right)=z \geq 0$ is the initial level and $\{M(t)\}_{t \geq 0}$ is a renewal process characterized by the sequence of (shifted) inter-arrival times $\left\{V_{i+1}\right\}_{i=1}^{\infty}$. Note that the process $\left\{Z_{D}(t)\right\}_{t \geq 0}$ starts with an upward jump of size $Y_{1}$ at time 0 , and it essentially behaves like $\left\{U_{D}(t)\right\}_{t \geq 0}$ but with the interval $\left[0, V_{1}\right)$ removed. With $T_{Z_{D}}=\inf \left\{t>0: Z_{D}(t)=0\right\}$ being the ruin time of $\left\{Z_{D}(t)\right\}_{t \geq 0}$, we also define $T_{Z_{D}}^{*}=\sum_{i=1}^{M\left(T_{Z_{D}}\right)+1} V_{i+1}$ and $\left|Z_{D}\left(T_{Z_{D}}^{*-}\right)\right|$ as analogues of $T_{U_{D}}^{*}$ and $\left|U_{D}\left(T_{U_{D}}^{*-}\right)\right|$ respectively, and the relationship $T_{Z_{D}}=T_{Z_{D}}^{*}-\left|Z_{D}\left(T_{Z_{D}}^{*-}\right)\right| / c$ holds. See Cheung (2012, Figure 2). The joint density of $\left(T_{Z_{D}}^{*},\left|Z\left(T_{Z_{D}}^{*-}\right)\right|\right)$ at $(t, y)$ pertaining to the process $(2.6)$ is denoted by $f_{Z_{D}}(t, y \mid z)$ for $y>0$ and $t>(z+y) / c$.

\subsection{Densities required to analyze the dual model}

Having defined various joint distributions for the processes $\left\{U_{D}(t)\right\}_{t \geq 0}$ and $\left\{Z_{D}(t)\right\}_{t \geq 0}$, we are ready to introduce related discounted densities which are needed in Section 3.2 concerning the dual model subject to exponential gains each with mean $1 / \mu$. By Cheung (2012, Equations (2.14) and (2.4)), the discounted density of $\left|U_{D}\left(T_{U_{D}}^{*-}\right)\right|$ at $y$ is given by

$$
h_{\delta, U_{D}}(y \mid u)=\frac{1}{c} e^{-\delta\left(\frac{u+y}{c}\right)} k\left(\frac{u+y}{c}\right)+\int_{\frac{u+y}{c}}^{\infty} e^{-\delta t} f_{U_{D}}(t, y \mid u) \mathrm{d} t, \quad y>0,
$$

while that of $\left|Z\left(T_{Z_{D}}^{*-}\right)\right|$ at $y$ is defined by

$$
h_{\delta, Z_{D}}(y \mid z)=\int_{\frac{z+y}{c}}^{\infty} e^{-\delta t} f_{Z_{D}}(t, y \mid z) \mathrm{d} t, \quad y>0 .
$$

In particular, at $z=0$, simplification of Cheung (2012, Equation (4.12)) yields

$$
h_{\delta, Z_{D}}(y \mid 0)=\mu e^{-\frac{\delta}{c} y} \int_{\frac{y}{c}}^{\infty} e^{-\left(R_{\delta}+\frac{\delta}{c}\right)(c t-y)} k(t) \mathrm{d} t,
$$


where $R_{\delta}$ is the unique non-negative root that satisfies the Lundberg's equation

$$
1-\widetilde{k}\left(c R_{\delta}+\delta\right) \frac{\mu}{\mu-R_{\delta}}=0 .
$$

Define $\kappa_{\delta}=\int_{0}^{\infty} h_{\delta, Z_{D}}(y \mid 0) \mathrm{d} y$. Note that $\kappa_{\delta}<1$ under the loading assumption in Section 3.2. Further define the proper density $\beta_{\delta}(y)=h_{\delta, Z_{D}}(y \mid 0) / \kappa_{\delta}$ for $y>0$, and the compound geometric density

$$
\alpha_{\delta}(y)=\sum_{n=1}^{\infty}\left(1-\kappa_{\delta}\right) \kappa_{\delta}^{n} \beta_{\delta}^{* n}(y), \quad y>0,
$$

where $\beta_{\delta}^{* n}(\cdot)$ represents the $n$-fold convolution density of $\beta_{\delta}(\cdot)$ with itself (and similar notation applies to the convolution of other densities). Then, from Equations (2.11) and (2.13) of Cheung (2012), the discounted densities defined in (2.8) and (2.7) can be fully characterized as

$$
\begin{aligned}
& h_{\delta, Z_{D}}(y \mid z)=\kappa_{\delta} \beta_{\delta}(z+y)+\frac{\kappa_{\delta}}{1-\kappa_{\delta}} \int_{0}^{z} \alpha_{\delta}(z-x) \beta_{\delta}(x+y) \mathrm{d} x . \\
& h_{\delta, U_{D}}(y \mid u)=\frac{1}{c} e^{-\delta\left(\frac{u+y}{c}\right)} k\left(\frac{u+y}{c}\right)+\int_{0}^{\frac{u}{c}} e^{-\delta t} h_{\delta, Z_{D}}(y \mid u-c t) k(t) \mathrm{d} t .
\end{aligned}
$$

Section 3.2 also requires the density of the time of ruin $T_{U_{S}}$ pertaining to the process $\left\{U_{S}(t)\right\}_{t \geq 0}$ in (1.2) with initial surplus $U_{S}(0)=u$. Under general inter-arrival times and exponential jumps, this density at $t$ is given by

$$
f_{U_{S}}(t \mid u)=e^{-\mu(u+c t)} \sum_{n=0}^{\infty} \frac{\mu^{n}(u+c t)^{n-1}}{n !}\left(u+\frac{c t}{n+1}\right) k^{*(n+1)}(t), \quad t>0 .
$$

See Borovkov and Dickson (2008, Equation (3)). Interested readers are also referred to Drekic and Willmot (2003), Dickson et al. (2005, Section 3.3) and Dickson and Li (2010, Section 4) when the inter-arrival times follow exponential, $\operatorname{Erlang}(n)$ and $\operatorname{Erlang}(2)$ distributions respectively.

\subsection{A density required to analyze the insurance model}

The exact solution to the joint density $f_{Z_{D}}(t, y \mid 0)$ will be required in Section 3.1 to study the insurance model with exponential claims. As demonstrated by a number of papers in the literature (e.g. Dickson and Willmot (2005)), the Lagrange's expansion theorem (also known as Lagrange's inversion theorem or Lagrange's implicit function theorem) plays a major role in obtaining probability densities involving the time component via inversion of appropriate Laplace transforms. The Lagrange's expansion theorem is now stated as follows for the sake of completeness. If

$$
v=w+\gamma \varphi(v)
$$

then for any analytic function $a(\cdot)$, one has

$$
a(v)=a(w)+\left.\sum_{n=1}^{\infty} \frac{\gamma^{n}}{n !} \frac{\partial^{n-1}}{\partial x^{n-1}}\left\{a^{\prime}(x)[\varphi(x)]^{n}\right\}\right|_{x=w} .
$$

Consider the process $\left\{Z_{D}(t)\right\}_{t \geq 0}$ defined via (2.6) when each jump $Y_{i}$ is exponential with mean $1 / \mu$ and the inter-arrival times $\left\{V_{i+1}\right\}_{i=1}^{\infty}$ have arbitrary common density $k(\cdot)$. For convenience, we 
define $b(x)=(1 / c) k(x / c)$ to be the density of a scaled inter-arrival time, and the corresponding Laplace transform is $\widetilde{b}(s)=\widetilde{k}(c s)$. Then the Lundberg's equation (2.10) which defines $R_{\delta}$ can be rewritten as

$$
R_{\delta}=\mu-\mu \widetilde{b}\left(R_{\delta}+\frac{\delta}{c}\right)
$$

Because of (2.8) and (2.9), we have the relationship

$$
\int_{\frac{y}{c}}^{\infty} e^{-\delta t} f_{Z_{D}}(t, y \mid 0) \mathrm{d} t=\mu e^{-\frac{\delta}{c} y} \int_{\frac{y}{c}}^{\infty} e^{-\left(R_{\delta}+\frac{\delta}{c}\right)(c t-y)} k(t) \mathrm{d} t .
$$

Our goal is to invert the Laplace transform on the right-hand side of (2.13) with respect to $\delta$ so as to obtain $f_{Z_{D}}(t, y \mid 0)$. By applying Lagrange's expansion theorem to the integrand on the right-hand side of (2.13) (with $a(v)=e^{-(v+\delta / c)(c t-y)}, v=R_{\delta}, w=\mu, \gamma=-\mu$ and $\varphi(v)=\widetilde{b}(v+\delta / c)$ ), we arrive at

$$
\begin{aligned}
\int_{\frac{y}{c}}^{\infty} e^{-\delta t} f_{Z_{D}}(t, y \mid 0) \mathrm{d} t= & \mu e^{-\frac{\delta}{c} y} \int_{\frac{y}{c}}^{\infty}\left\{e^{-\left(\mu+\frac{\delta}{c}\right)(c t-y)}\right. \\
& \left.+\left.\sum_{n=1}^{\infty} \frac{(-\mu)^{n}}{n !} \frac{\partial^{n-1}}{\partial x^{n-1}}\left((y-c t) e^{-\left(x+\frac{\delta}{c}\right)(c t-y)} \int_{0}^{\infty} e^{-\left(x+\frac{\delta}{c}\right) z} b^{* n}(z) \mathrm{d} z\right)\right|_{x=\mu}\right\} k(t) \mathrm{d} t
\end{aligned}
$$

where we have used the fact that $[\widetilde{b}(s)]^{n}=\int_{0}^{\infty} e^{-s z} b^{* n}(z) \mathrm{d} z$. While the first term in (2.14) is given by

$$
\mu e^{-\frac{\delta}{c} y} \int_{\frac{y}{c}}^{\infty} e^{-\left(\mu+\frac{\delta}{c}\right)(c t-y)} k(t) \mathrm{d} t=\int_{\frac{y}{c}}^{\infty} e^{-\delta t}\left(\mu e^{-\mu(c t-y)} k(t)\right) \mathrm{d} t,
$$

the second term can be simplified as

$$
\begin{aligned}
& \left.\mu e^{-\frac{\delta}{c} y} \int_{\frac{y}{c}}^{\infty} \sum_{n=1}^{\infty} \frac{(-\mu)^{n}}{n !} \frac{\partial^{n-1}}{\partial x^{n-1}}\left((y-c t) e^{-\left(x+\frac{\delta}{c}\right)(c t-y)} \int_{0}^{\infty} e^{-\left(x+\frac{\delta}{c}\right) z} b^{* n}(z) \mathrm{d} z\right)\right|_{x=\mu} k(t) \mathrm{d} t \\
= & \left.\mu e^{-\frac{\delta}{c} y} \int_{\frac{y}{c}}^{\infty} \sum_{n=1}^{\infty} \frac{(-\mu)^{n}}{n !} \frac{\partial^{n-1}}{\partial x^{n-1}}\left((y-c t) \int_{0}^{\infty} e^{-\left(x+\frac{\delta}{c}\right)(z+c t-y)} b^{* n}(z) \mathrm{d} z\right)\right|_{x=\mu} k(t) \mathrm{d} t \\
= & \mu e^{-\frac{\delta}{c} y} \int_{\frac{y}{c}}^{\infty} \sum_{n=1}^{\infty} \frac{(-\mu)^{n}}{n !}\left((y-c t)(-1)^{n-1} \int_{0}^{\infty}(z+c t-y)^{n-1} e^{-\left(\mu+\frac{\delta}{c}\right)(z+c t-y)} b^{* n}(z) \mathrm{d} z\right) k(t) \mathrm{d} t \\
= & \int_{\frac{y}{c}}^{\infty} \int_{c t}^{\infty} \sum_{n=1}^{\infty} \frac{\mu^{n+1}}{n !} e^{-\frac{\delta}{c} z}(c t-y)(z-y)^{n-1} e^{-\mu(z-y)} b^{* n}(z-c t) k(t) \mathrm{d} z \mathrm{~d} t \\
= & \int_{y}^{\infty} \int_{\frac{y}{c}}^{\frac{z}{c}} \sum_{n=1}^{\infty} \frac{\mu^{n+1}}{n !} e^{-\frac{\delta}{c} z}(c t-y)(z-y)^{n-1} e^{-\mu(z-y)} b^{* n}(z-c t) k(t) \mathrm{d} t \mathrm{~d} z \\
= & \int_{\frac{y}{c}}^{\infty} \int_{\frac{y}{c}}^{z} \sum_{n=1}^{\infty} \frac{\mu^{n+1}}{n !} e^{-\delta z}(c t-y)(c z-y)^{n-1} e^{-\mu(c z-y)} b^{* n}(c z-c t) k(t) c \mathrm{~d} t \mathrm{~d} z \\
= & \int_{\frac{y}{c}}^{\infty} e^{-\delta t}\left(\mu e^{-\mu(c t-y)} \sum_{n=1}^{\infty} \frac{\mu^{n}}{n !}(c t-y)^{n-1} \int_{\frac{y}{c}}^{t}(c z-y) k^{* n}(t-z) k(z) \mathrm{d} z\right) \mathrm{d} t .
\end{aligned}
$$


It follows from substitution of the above two expressions into (2.14) along with the uniqueness of the Laplace transform that, for $y>0$ and $t>y / c$,

$$
f_{Z_{D}}(t, y \mid 0)=\mu e^{-\mu(c t-y)}\left(k(t)+\sum_{n=1}^{\infty} \frac{\mu^{n}}{n !}(c t-y)^{n-1} \int_{\frac{y}{c}}^{t}(c z-y) k^{* n}(t-z) k(z) \mathrm{d} z\right) .
$$

\section{Laplace transform of Parisian ruin time}

\subsection{Sparre Andersen insurance model with exponential claims}

We start with the Sparre Andersen insurance risk model $\left\{U_{S}(t)\right\}_{t \geq 0}$ in (1.2) under exponential claim amounts and arbitrary interclaim times. The positive security loading condition $c \mathbb{E}\left[V_{1}\right]>\mathbb{E}\left[Y_{1}\right]=1 / \mu$ is assumed to hold, so that $\left\{U_{S}(t)\right\}_{t \geq 0}$ drifts to infinity in the long run and the ruin probability is strictly less than 1 (e.g. Prabhu (1998, Part I, Theorems 2 and 7)).

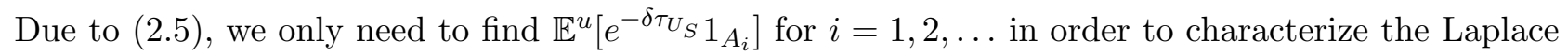
transform of the Parisian ruin time $\psi_{\delta, U_{S}}(u)$. Using the representations of the Parisian ruin time $(2.3)$ and the set $A_{i}$ in (2.4), it is immediate that

$$
\mathbb{E}^{u}\left[e^{-\delta \tau_{U_{S}}} 1_{A_{1}}\right]=\mathbb{E}^{u}\left[e^{-\delta\left(L_{+, 1}+d\right)} 1_{B_{1} \cap C_{1}^{c}}\right]=\mathbb{E}^{u}\left[\left(e^{-\delta L_{+, 1}} 1_{B_{1}}\right)\left(e^{-\delta d} 1_{C_{1}^{c}}\right)\right],
$$

and for $i=2,3, \ldots$,

$$
\begin{aligned}
\mathbb{E}^{u}\left[e^{\left.-\delta \tau_{U_{S}} 1_{A_{i}}\right]}\right. & =\mathbb{E}^{u}\left[e^{-\delta\left(\sum_{j=1}^{i} L_{+, j}+\sum_{j=1}^{i-1} L_{-, j}+d\right)} 1_{\left(\bigcap_{j=1}^{i} B_{j}\right) \cap\left(\bigcap_{j=1}^{i-1} C_{j}\right) \cap C_{i}^{c}}\right] \\
& =\mathbb{E}^{u}\left[e^{-\delta L_{+, 1}} 1_{B_{1}}\left(\prod_{j=1}^{i-1} e^{-\delta\left(L_{-, j}+L_{+, j+1}\right)} 1_{C_{j} \cap B_{j+1}}\right) e^{-\delta d_{1}} 1_{C_{i}^{c}}\right] .
\end{aligned}
$$

Note that the final expression indeed also holds true for $i=1$. Under exponential claims, it is well known (e.g. Willmot (2007, Example 3.1)) that whenever the surplus process $\left\{U_{S}(t)\right\}_{t \geq 0}$ drops below zero at time $\tau_{U_{S}}^{-, j}$ (see Figure 1), the resulting amount of shortfall follows the same exponential distribution with mean $1 / \mu$. This along with the renewal property of $\{N(t)\}_{t \geq 0}$ implies that the excursions for the process $\left\{U_{S}(t)\right\}_{t \geq 0}$ in the time intervals $\left[\tau_{U_{S}}^{-, j-1}, \tau_{U_{S}}^{-, j}\right)$ for $j=1,2, \ldots$ are all independent. In particular, given $L_{+, 1}$ and the event $B_{1}$, the only impact on the events subsequent to time $\tau_{U_{S}}^{-, 1}$ is the exponential amount of shortfall at time $\tau_{U_{S}}^{-, 1}$. Along the same line of logic, for each fixed $j=2,3, \ldots$, given $L_{+, 1}, B_{1}, L_{-, 1}$, $C_{1}, L_{+, 2}, B_{2} \ldots, L_{-, j-1}, C_{j-1}, L_{+, j}, B_{j}$, the amount of shortfall at time $\tau_{U_{S}^{-}}^{-, j}$ is exponentially distributed, and this is the only impact on events subsequent to time $\tau_{U_{S}}^{-, j}$. Because the events in the time intervals $\left[\tau_{U_{S}}^{-, j}, \tau_{U_{S}}^{-, j+1}\right)$ for $j=1,2, \ldots$ follow the same probability law, we have that, for $i=1,2, \ldots$,

$$
\mathbb{E}^{u}\left[e^{-\delta \tau_{U_{S}}} 1_{A_{i}}\right]=\mathbb{E}^{u}\left[e^{-\delta L_{+, 1}} 1_{B_{1}}\right]\left(\mathbb{E}^{*}\left[e^{-\delta\left(L_{-, 1}+L_{+, 2}\right)} 1_{C_{1} \cap B_{2}}\right]\right)^{i-1} \mathbb{E}^{*}\left[e^{-\delta d} 1_{C_{1}^{c}}\right],
$$

where the expectation $\mathbb{E}^{*}$ is conditional on that $\left|U_{S}\left(\tau_{U_{S}}^{-, 1}\right)\right|$ is exponential with mean $1 / \mu$.

From (3.1), it suffices to evaluate the three expectations $\mathbb{E}^{u}\left[e^{-\delta L_{+, 1}} 1_{B_{1}}\right], \mathbb{E}^{*}\left[e^{-\delta\left(L_{-, 1}+L_{+, 2}\right)} 1_{C_{1} \cap B_{2}}\right]$ and $\mathbb{E}^{*}\left[e^{-\delta d} 1_{C_{1}^{c}}\right]$. The first expectation is simply the Laplace transform of the time of ruin under the traditional definition, which is given by e.g. Willmot (2007, Equation (3.15)) as

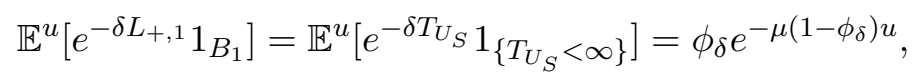


where $\phi_{\delta}$ (such that $0<\phi_{\delta}<1$ ) satisfies the Lundberg's equation

$$
\phi_{\delta}=\widetilde{k}\left(\delta+c \mu\left(1-\phi_{\delta}\right)\right) .
$$

Note that $R_{\delta}$ defined via $(2.10)$ is related to $\phi_{\delta}$ by $R_{\delta}=\mu\left(1-\phi_{\delta}\right)$.
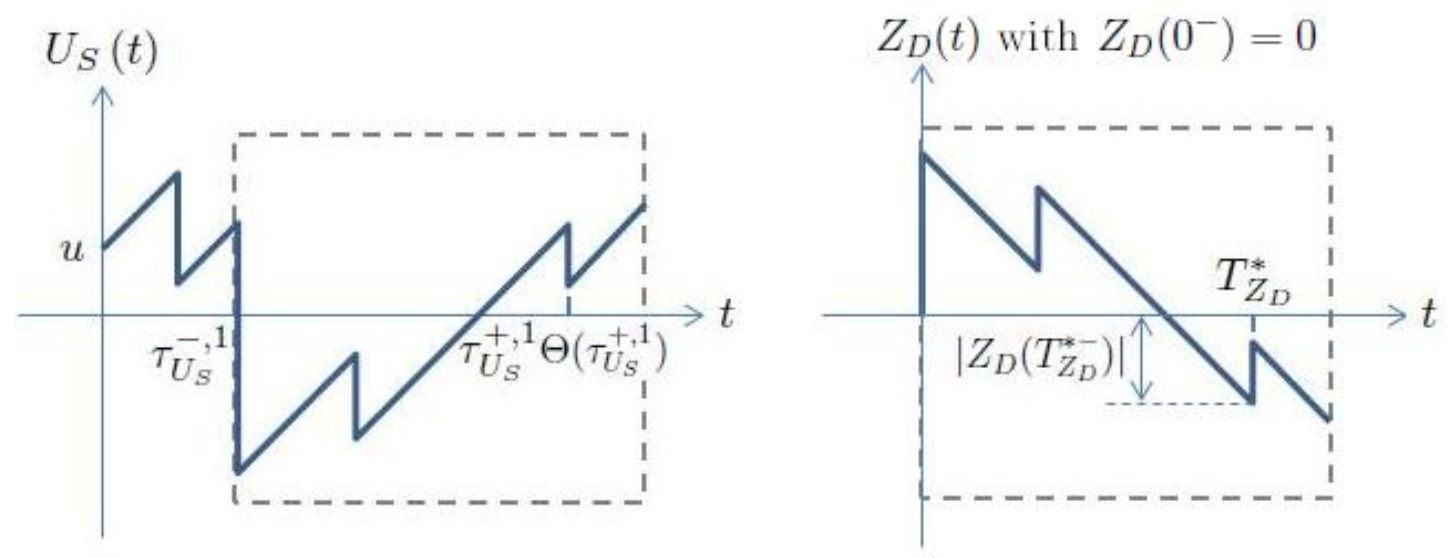

Figure 3: Relationship between $\left\{U_{S}(t)\right\}_{t \geq \tau_{U}^{-, 1}}$ and $\left\{Z_{D}(t)\right\}_{t \geq 0}$

The key to evaluating the two remaining expectations is to recognize that the law of the reflection of the process $\left\{U_{S}(t)\right\}_{t \geq \tau_{U_{S}}^{-, 1}}$ (conditional on $\left.\tau_{U_{S}^{-, 1}}^{-,}<\infty\right)$ is actually identical to that of the process $\left\{Z_{D}(t)\right\}_{t \geq 0}$ with initial condition $Z_{D}\left(0^{-}\right)=0$. See Figure 3 . Note that because of the loading condition $c \mathbb{E}\left[V_{1}\right]>\mathbb{E}\left[Y_{1}\right]=1 / \mu$, given $\tau_{U_{S}}^{-, 1}<\infty$ the recovery of $\left\{U_{S}(t)\right\}_{t \geq \tau_{U_{S}}^{-, 1}}$ occurs almost surely, i.e. $T_{Z_{D}}<\infty$ almost surely for $\left\{Z_{D}(t)\right\}_{t \geq 0}$. Define $\Theta(s)=\inf \left\{t>s: N\left(t^{-}\right)+1=N(t)\right\}$ to be the first time when a jump occurs after the time point $s$. The following observations can be made.

- The joint distribution of $\left(\Theta\left(\tau_{U_{S}}^{+, 1}\right)-\tau_{U_{S}}^{-, 1}, U_{S}\left(\Theta\left(\tau_{U_{S}}^{+, 1}\right)^{-}\right)\right)$given $\tau_{U_{S}}^{-, 1}<\infty$ is the same as that of $\left(T_{Z_{D}}^{*},\left|Z\left(T_{Z_{D}}^{*-}\right)\right|\right)$ under $Z_{D}\left(0^{-}\right)=0$, whose joint density $f_{Z_{D}}(t, y \mid 0)$ is given in $(2.15)$.

- The relationship $L_{-, 1}=\Theta\left(\tau_{U_{S}}^{+, 1}\right)-\tau_{U_{S}}^{-, 1}-U_{S}\left(\Theta\left(\tau_{U_{S}}^{+, 1}\right)^{-}\right) / c$ holds.

- Due to the renewal property of $\{N(t)\}_{t \geq 0}$, the law of $\left\{U_{S}(t)\right\}_{t \geq \Theta\left(\tau_{U_{S}}^{+, 1}\right)}$ only depends on the surplus level $U_{S}\left(\Theta\left(\tau_{U_{S}}^{+, 1}\right)\right)=U_{S}\left(\Theta\left(\tau_{U_{S}}^{+, 1}\right)^{-}\right)-Y_{N\left(\Theta\left(\tau_{U_{S}}^{+, 1}\right)\right)}$, where $Y_{N\left(\Theta\left(\tau_{U_{S}}^{+, 1}\right)\right)}$ is the exponential jump that occurs at time $\Theta\left(\tau_{U_{S}}^{+, 1}\right)$ which is independent of $U_{S}\left(\Theta\left(\tau_{U_{S}}^{+, 1}\right)^{-}\right)$.

- If $Y_{N\left(\Theta\left(\tau_{U_{S}}^{+, 1}\right)\right)}>U_{S}\left(\Theta\left(\tau_{U_{S}}^{+, 1}\right)^{-}\right)$then $\tau_{U_{S}}^{-, 2}=\Theta\left(\tau_{U_{S}}^{+, 1}\right)$. On the other hand, if $Y_{N\left(\Theta\left(\tau_{U_{S}}^{+, 1}\right)\right)} \leq U_{S}\left(\Theta\left(\tau_{U_{S}}^{+, 1}\right)^{-}\right)$, then the process $\left\{U_{S}(t)\right\}_{t \geq \Theta\left(\tau_{U_{S}}^{+, 1}\right)}$ restarts at time $\Theta\left(\tau_{U_{S}}^{+, 1}\right)$ with $\tau_{U_{S}}^{-, 2}-\Theta\left(\tau_{U_{S}}^{+, 1}\right)$ having the same distribution as the time of ruin of the process $\left\{\underline{U}_{S}(t)\right\}_{t \geq 0}$ under $\underline{U}_{S}(0)=U_{S}\left(\Theta\left(\tau_{U_{S}}^{+, 1}\right)^{-}\right)-Y_{N\left(\Theta\left(\tau_{U_{S}}^{+, 1}\right)\right)}$. (Here $\left\{\underline{U}_{S}(t)\right\}_{t \geq 0}$ is an independent process with the same law as $\left\{U_{S}(t)\right\}_{t \geq 0}$.)

Hence, by conditioning on $Y_{N\left(\Theta\left(\tau_{U_{S}}^{+, 1}\right)\right)}$ along with the use of $(3.2)$ and the density $f_{Z_{D}}(t, y \mid 0)$, we arrive at

$$
\mathbb{E}^{*}\left[e^{-\delta\left(L_{-, 1}+L_{+, 2}\right)} 1_{C_{1} \cap B_{2}}\right]=\mathbb{E}^{*}\left[e^{-\delta\left[\left(\Theta\left(\tau_{U_{S}}^{+, 1}\right)-\tau_{U_{S}}^{-, 1}\right)+\left(\tau_{U_{S}}^{-, 2}-\Theta\left(\tau_{U_{S}}^{+, 1}\right)\right)\right]} 1_{\left\{L_{-, 1}<d\right\} \cap\left\{L_{+, 2}<\infty\right\}}\right]
$$




$$
\begin{aligned}
= & \int_{0}^{\infty} \int_{0}^{y}\left(\int_{\frac{y}{c}}^{\frac{y}{c}+d} e^{-\delta t} f_{Z_{D}}(t, y \mid 0) \mathrm{d} t\right)\left(\mu e^{-\mu x}\right)\left(\phi_{\delta} e^{-\mu\left(1-\phi_{\delta}\right)(y-x)}\right) \mathrm{d} x \mathrm{~d} y \\
& +\int_{0}^{\infty} \int_{y}^{\infty}\left(\int_{\frac{y}{c}}^{\frac{y}{c}+d} e^{-\delta t} f_{Z_{D}}(t, y \mid 0) \mathrm{d} t\right)\left(\mu e^{-\mu x}\right) \mathrm{d} x \mathrm{~d} y \\
= & \int_{0}^{\infty} e^{-\mu\left(1-\phi_{\delta}\right) y} \int_{\frac{y}{c}}^{\frac{y}{c}+d} e^{-\delta t} f_{Z_{D}}(t, y \mid 0) \mathrm{d} t \mathrm{~d} y .
\end{aligned}
$$

Similar reasoning leads us to

$$
\mathbb{E}^{*}\left[e^{-\delta d} 1_{C_{1}^{c}}\right]=e^{-\delta d} \mathbb{P}\left\{T_{Z_{D}}^{*}-\frac{\left|Z\left(T_{Z_{D}}^{*-}\right)\right|}{c} \geq d \mid Z_{D}\left(0^{-}\right)=0\right\}=e^{-\delta d} \int_{0}^{\infty} \int_{\frac{y}{c}+d}^{\infty} f_{Z_{D}}(t, y \mid 0) \mathrm{d} t \mathrm{~d} y .
$$

To summarize, using (3.1), the Laplace transform of the Parisian ruin time in (2.5) reduces to

$$
\psi_{\delta, U_{S}}(u)=\frac{\mathbb{E}^{u}\left[e^{-\delta L_{+, 1}} 1_{B_{1}}\right] \mathbb{E}^{*}\left[e^{-\delta d_{1}} 1_{C_{1}^{c}}\right]}{1-\mathbb{E}^{*}\left[e^{-\delta\left(L_{-, 1}+L_{+, 2}\right)} 1_{C_{1} \cap B_{2}}\right]},
$$

where the components are given by (3.2)-(3.4). It is interesting to note that (3.5) simply equals the Laplace transform of the traditional ruin time (3.2) (which is an exponential function in $u$ ) multiplied by a constant term that is independent of $u$.

Remark 1 Define, on the event of Parisian ruin, $Q^{*}=\sup \left\{n \in\{0\} \cup \mathbb{N}: \tau_{U_{S}}^{-, n+1}<\tau_{U_{S}}\right\}$ to be the number of periods of negative surplus before Parisian ruin (excluding the one in which Parisian ruin occurs). Note that the set $A_{i}$ defined in (2.4) is equivalent to $A_{i}=\left\{\tau_{U_{S}}<\infty\right\} \cap\left\{Q^{*}=i-1\right\}$ for $i=1,2, \ldots$. Let $\mathbb{P}^{u}$ be the probability measure under the initial condition $U_{S}(0)=u$, and $\mathbb{P}^{*}$ be the probability conditional on that $\left|U_{S}\left(\tau_{U_{S}}^{-, 1}\right)\right|$ is exponential with mean $1 / \mu$. Use of (3.1) and (3.5) with $\delta=0$ yields

$$
\mathbb{P}^{u}\left\{Q^{*}=i \mid \tau_{U_{S}}<\infty\right\}=\frac{\mathbb{P}^{u}\left\{A_{i+1}\right\}}{\psi_{0, U_{S}}(u)}=\left(\mathbb{P}^{*}\left\{C_{1} \cap B_{2}\right\}\right)^{i}\left(1-\mathbb{P}^{*}\left\{C_{1} \cap B_{2}\right\}\right), \quad i=0,1, \ldots
$$

In other words, conditional on the event of Parisian ruin, $Q^{*}$ follows the same geometric distribution regardless of the initial surplus level $u \geq 0$.

\subsection{Dual Sparre Andersen model with exponential gains}

In this subsection, we look at the dual Sparre Andersen risk process $\left\{U_{D}(t)\right\}_{t \geq 0}$ in which the gains $\left\{Y_{i}\right\}_{i=1}^{\infty}$ are exponential with mean $1 / \mu$ and the inter-arrival times $\left\{V_{i}\right\}_{i=1}^{\infty}$ follow an arbitrary distribution. It is assumed that the loading condition $1 / \mu>c \mathbb{E}\left[V_{1}\right]$ is satisfied.

As in Section 3.1, we need to determine $\mathbb{E}^{u}\left[e^{-\delta \tau_{U_{D}} 1_{A_{i}}}\right]$ for $i=1,2, \ldots$, which can be done by partitioning the sample path in Figure 2 into independent segments such that the corresponding Laplace transforms can be multiplied together. Application of (2.3) and (2.4) results in

$$
\mathbb{E}^{u}\left[e^{-\delta \tau_{U_{D}}} 1_{A_{1}}\right]=\mathbb{E}^{u}\left[e^{-\delta\left(L_{+, 1}+d\right)} 1_{B_{1} \cap C_{1}^{c}}\right],
$$

and, for $i=2,3, \ldots$,

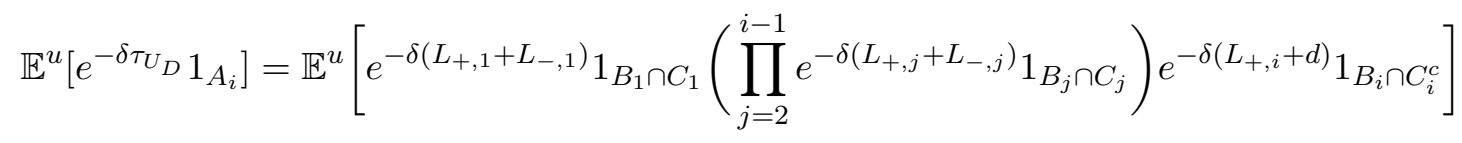




$$
=\mathbb{E}^{u}\left[e^{-\delta\left(L_{+, 1}+L_{-, 1}\right)} 1_{B_{1} \cap C_{1}}\right]\left(\mathbb{E}^{*}\left[e^{-\delta\left(L_{+, 2}+L_{-, 2}\right)} 1_{B_{2} \cap C_{2}}\right]\right)^{i-2} \mathbb{E}^{*}\left[e^{-\delta\left(L_{+, 2}+d\right)} 1_{B_{2} \cap C_{2}^{c}}\right],
$$

where $\mathbb{E}^{*}$ is now the conditional expectation given that $U_{D}\left(\tau_{U_{D}}^{+, 1}\right)$ is exponential with mean $1 / \mu$, i.e. from time $\tau_{U_{D}}^{+, 1}$ onward the process $\left\{U_{D}(t)\right\}_{t \geq \tau_{U_{D}}^{+, 1}}$ behaves like $\left\{Z_{D}(t)\right\}_{t \geq 0}$ with $Z_{D}\left(0^{-}\right)=0$. The second equality above can be obtained as follows. For each fixed $j=1,2, \ldots$, given $L_{+, 1}, B_{1}, L_{-, 1}, C_{1}, \ldots, L_{+, j}$, $B_{j}, L_{-, j}, C_{j}$, the only impact on events subsequent to time $\tau_{U_{D}}^{+, j}$ is the exponential jump $Y_{N\left(\tau_{U}^{+, j}\right)}$ at time $\tau_{U_{D}}^{+, j}$. By the memoryless property, the resulting overshoot above zero at time $\tau_{U_{D}}^{+, j}$ follows the same exponential distribution with mean $1 / \mu$. The excursions of the process $\left\{U_{D}(t)\right\}_{t \geq 0}$ in $\left[\tau_{U_{D}}^{+, j-1}, \tau_{U_{D}}^{+, j}\right)$ are independent for all $j=1,2, \ldots$, but they follow the same probability law only for $j=2,3, \ldots$ These explain (3.7).

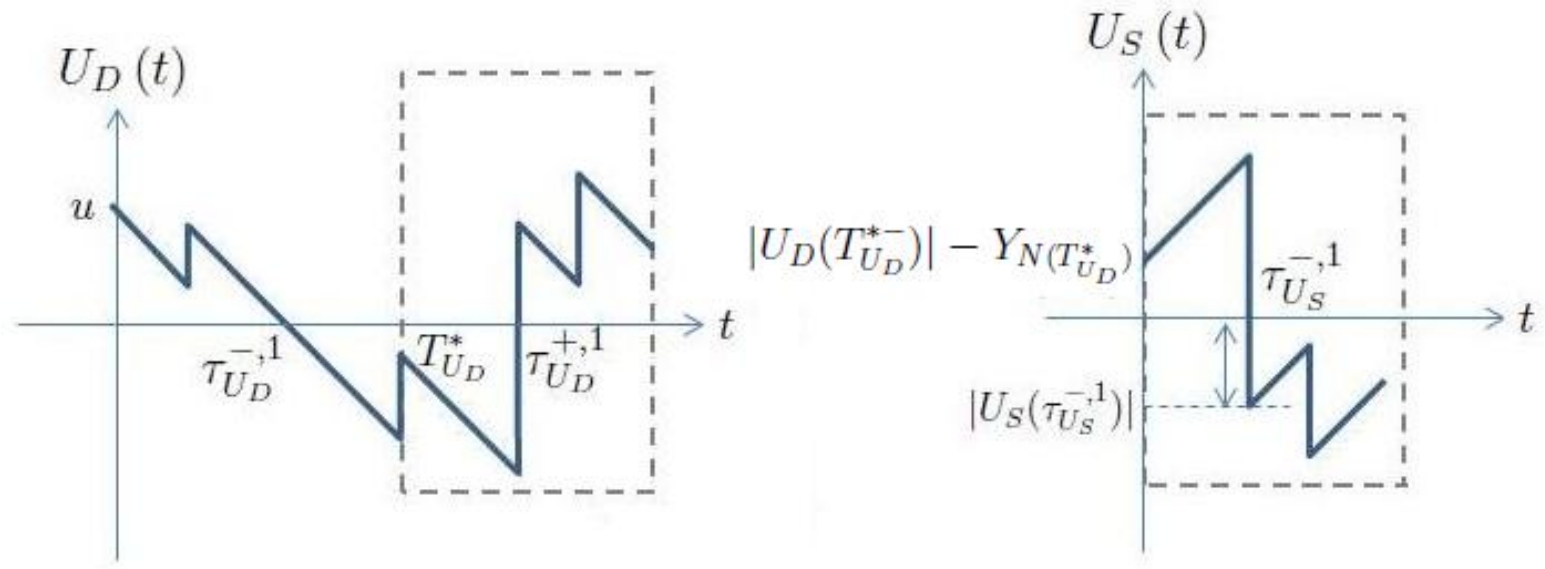

Figure 4: Relationship between $\left\{U_{D}(t)\right\}_{t \geq T_{U_{D}}^{*}}$ and $\left\{U_{S}(t)\right\}_{t \geq 0}$

It is clear from (3.6) and (3.7) that it remains to determine the four expectations $\mathbb{E}^{u}\left[e^{-\delta\left(L_{+, 1}+d\right)} 1_{B_{1} \cap C_{1}^{c}}\right]$, $\mathbb{E}^{u}\left[e^{-\delta\left(L_{+, 1}+L_{-, 1}\right)} 1_{B_{1} \cap C_{1}}\right], \mathbb{E}^{*}\left[e^{-\delta\left(L_{+, 2}+L_{-, 2}\right)} 1_{B_{2} \cap C_{2}}\right]$ and $\mathbb{E}^{*}\left[e^{-\delta\left(L_{+, 2}+d\right)} 1_{B_{2} \cap C_{2}^{c}}\right]$. For the first two expectations, we note that in the case where the jump $Y_{N\left(T_{U_{D}}^{*}\right)}$ at time $T_{U_{D}}^{*}$ does not result in an overshoot above zero, the reflection of the process $\left\{U_{D}(t)\right\}_{t \geq T_{U_{D}}^{*}}$ is identical in law to $\left\{U_{S}(t)\right\}_{t \geq 0}$ with the initial level $U_{S}(0)=\left|U_{D}\left(T_{U_{D}}^{*-}\right)\right|-Y_{N\left(T_{U_{D}}^{*}\right)}$. See Figure 4. Similar connections are applicable to $\left\{Z_{D}(t)\right\}_{t \geq T_{Z_{D}}^{*}}$ and $\left\{U_{S}(t)\right\}_{t \geq 0}$, and these will be needed to evaluate the expectation $\mathbb{E}^{*}$. In order to use similar conditioning arguments leading to (3.3), we shall apply the densities $f_{U_{D}}(t, y \mid u), f_{U_{S}}(t \mid u)$ and $f_{Z_{D}}(t, y \mid 0)$ defined in Section 2. Consolidating the above observations, $\mathbb{E}^{u}\left[e^{-\delta\left(L_{+, i}+d\right)} 1_{B_{1} \cap C_{1}^{c}}\right]$ is first evaluated as

$$
\begin{aligned}
\mathbb{E}^{u}\left[e^{-\delta\left(L_{+, 1}+d\right)} 1_{B_{1} \cap C_{1}^{c}}\right]= & \int_{c d}^{\infty} e^{-\delta\left(\frac{u}{c}+d\right)}\left(\frac{1}{c} k\left(\frac{u+y}{c}\right)\right) \mathrm{d} y+\int_{c d}^{\infty} \int_{\frac{u+y}{c}}^{\infty} e^{-\delta\left(t-\frac{y}{c}+d\right)} f_{U_{D}}(t, y \mid u) \mathrm{d} t \mathrm{~d} y \\
& +\int_{0}^{c d} \int_{0}^{y} \int_{d-\frac{y}{c}}^{\infty} e^{-\delta\left(\frac{u}{c}+d\right)}\left(\frac{1}{c} k\left(\frac{u+y}{c}\right)\right)\left(\mu e^{-\mu x}\right) f_{U_{S}}(v \mid y-x) \mathrm{d} v \mathrm{~d} x \mathrm{~d} y \\
& +\int_{0}^{c d} \int_{\frac{u+y}{c}}^{\infty} \int_{0}^{y} \int_{d-\frac{y}{c}}^{\infty} e^{-\delta\left(t-\frac{y}{c}+d\right)} f_{U_{D}}(t, y \mid u)\left(\mu e^{-\mu x}\right) f_{U_{S}}(v \mid y-x) \mathrm{d} v \mathrm{~d} x \mathrm{~d} t \mathrm{~d} y \\
= & \int_{c d}^{\infty} e^{-\delta\left(d-\frac{y}{c}\right)} h_{\delta, U_{D}}(y \mid u) \mathrm{d} y
\end{aligned}
$$




$$
+\int_{0}^{c d} \int_{0}^{y} \int_{d-\frac{y}{c}}^{\infty} e^{-\delta\left(d-\frac{y}{c}\right)} h_{\delta, U_{D}}(y \mid u)\left(\mu e^{-\mu x}\right) f_{U_{S}}(v \mid y-x) \mathrm{d} v \mathrm{~d} x \mathrm{~d} y,
$$

where the last equality follows from $(2.7)$. The expectation $\mathbb{E}^{*}\left[e^{-\delta\left(L_{+, 2}+d\right)} 1_{B_{2} \cap C_{2}^{c}}\right]$ can be derived in a similar manner with the use of (2.8), giving

$$
\begin{aligned}
\mathbb{E}^{*}\left[e^{-\delta\left(L_{+, 2}+d\right)} 1_{B_{2} \cap C_{2}}\right]= & \int_{c d}^{\infty} \int_{\frac{y}{c}}^{\infty} e^{-\delta\left(t-\frac{y}{c}+d\right)} f_{Z_{D}}(t, y \mid 0) \mathrm{d} t \mathrm{~d} y \\
& +\int_{0}^{c d} \int_{\frac{y}{c}}^{\infty} \int_{0}^{y} \int_{d-\frac{y}{c}}^{\infty} e^{-\delta\left(t-\frac{y}{c}+d\right)} f_{Z_{D}}(t, y \mid 0)\left(\mu e^{-\mu x}\right) f_{U_{S}}(v \mid y-x) \mathrm{d} v \mathrm{~d} x \mathrm{~d} t \mathrm{~d} y \\
= & \int_{c d}^{\infty} e^{-\delta\left(d-\frac{y}{c}\right)} h_{\delta, Z_{D}}(y \mid 0) \mathrm{d} y \\
& +\int_{0}^{c d} \int_{0}^{y} \int_{d-\frac{y}{c}}^{\infty} e^{-\delta\left(d-\frac{y}{c}\right)} h_{\delta, Z_{D}}(y \mid 0)\left(\mu e^{-\mu x}\right) f_{U_{S}}(v \mid y-x) \mathrm{d} v \mathrm{~d} x \mathrm{~d} y
\end{aligned}
$$

Upon integration over another appropriate region, $\mathbb{E}^{u}\left[e^{-\delta\left(L_{+, 1}+L_{-, 1}\right)} 1_{B_{1} \cap C_{1}}\right]$ is found to be

$$
\begin{aligned}
\mathbb{E}^{u}\left[e^{-\delta\left(L_{+, 1}+L_{-, 1}\right)} 1_{B_{1} \cap C_{1}}\right]= & \int_{0}^{c d} \int_{y}^{\infty} e^{-\delta\left(\frac{u+y}{c}\right)}\left(\frac{1}{c} k\left(\frac{u+y}{c}\right)\right)\left(\mu e^{-\mu x}\right) \mathrm{d} x \mathrm{~d} y \\
& +\int_{0}^{c d} \int_{\frac{u+y}{c}}^{\infty} \int_{y}^{\infty} e^{-\delta t} f_{U_{D}}(t, y \mid u)\left(\mu e^{-\mu x}\right) \mathrm{d} x \mathrm{~d} t \mathrm{~d} y \\
& +\int_{0}^{c d} \int_{0}^{y} \int_{0}^{d-\frac{y}{c}} e^{-\delta\left(\frac{u+y}{c}+v\right)}\left(\frac{1}{c} k\left(\frac{u+y}{c}\right)\right)\left(\mu e^{-\mu x}\right) f_{U_{S}}(v \mid y-x) \mathrm{d} v \mathrm{~d} x \mathrm{~d} y \\
& +\int_{0}^{c d} \int_{\frac{u+y}{c}}^{\infty} \int_{0}^{y} \int_{0}^{d-\frac{y}{c}} e^{-\delta(t+v)} f_{U_{D}}(t, y \mid u)\left(\mu e^{-\mu x}\right) f_{U_{S}}(v \mid y-x) \mathrm{d} v \mathrm{~d} x \mathrm{~d} t \mathrm{~d} y \\
= & \int_{0}^{c d} h_{\delta, U_{D}}(y \mid u)\left(e^{-\mu y}\right) \mathrm{d} y \\
& +\int_{0}^{c d} \int_{0}^{y} \int_{0}^{d-\frac{y}{c}} e^{-\delta v} h_{\delta, U_{D}}(y \mid u)\left(\mu e^{-\mu x}\right) f_{U_{S}}(v \mid y-x) \mathrm{d} v \mathrm{~d} x \mathrm{~d} y
\end{aligned}
$$

while $\mathbb{E}^{*}\left[e^{-\delta\left(L_{+, 2}+L_{-, 2}\right)} 1_{B_{2} \cap C_{2}}\right]$ is similarly given by

$$
\begin{aligned}
\mathbb{E}^{*}\left[e^{-\delta\left(L_{+, 2}+L_{-, 2}\right)} 1_{B_{2} \cap C_{2}}\right]= & \int_{0}^{c d} h_{\delta, Z_{D}}(y \mid 0)\left(e^{-\mu y}\right) \mathrm{d} y \\
& +\int_{0}^{c d} \int_{0}^{y} \int_{0}^{d-\frac{y}{c}} e^{-\delta v} h_{\delta, Z_{D}}(y \mid 0)\left(\mu e^{-\mu x}\right) f_{U_{S}}(v \mid y-x) \mathrm{d} v \mathrm{~d} x \mathrm{~d} y .
\end{aligned}
$$

It is instructive to note that although our intermediate steps involve three densities $f_{U_{D}}(t, y \mid u), f_{U_{S}}(t \mid u)$ and $f_{Z_{D}}(t, y \mid 0)$, the dependence on $f_{U_{D}}(t, y \mid u)$ and $f_{Z_{D}}(t, y \mid 0)$ of the final expressions in (3.8)-(3.11) only appears via the discounted densities $h_{\delta, U_{D}}(y \mid u)$ and $h_{\delta, Z_{D}}(y \mid 0)$. These two discounted densities are given in (2.11) and (2.9) respectively, and are much easier to evaluate compared to $f_{U_{D}}(t, y \mid u)$ and $f_{Z_{D}}(t, y \mid 0)$. The remaining density $f_{U_{S}}(t \mid u)$ can be computed by $(2.12)$.

With the four expectations appearing in (3.6) and (3.7) determined above, the Laplace transform of the Parisian ruin time in $(2.5)$ reduces to

$$
\psi_{\delta, U_{D}}(u)=\mathbb{E}^{u}\left[e^{-\delta\left(L_{+, 1}+d\right)} 1_{B_{1} \cap C_{1}^{c}}\right]+\frac{\mathbb{E}^{u}\left[e^{-\delta\left(L_{+, 1}+L_{-, 1}\right)} 1_{B_{1} \cap C_{1}}\right] \mathbb{E}^{*}\left[e^{-\delta\left(L_{+, 2}+d\right)} 1_{B_{2} \cap C_{2}^{c}}\right]}{1-\mathbb{E}^{*}\left[e^{-\delta\left(L_{+, 2}+L_{-, 2}\right)} 1_{B_{2} \cap C_{2}}\right]} .
$$




\section{Number of periods of negative surplus}

In this section, we look at the joint distribution of the numbers of periods of negative surplus that are of duration more than $d$ and less than $d$. This distribution is easily obtainable using the techniques and intermediate functions introduced in Section 3. For illustrations, we only study the Sparre Andersen insurance risk model (1.2) under the distributional assumptions in Section 3.1, i.e. the inter-arrival times are general while the claims are exponential, and the loading condition $c \mathbb{E}\left[V_{1}\right]>1 / \mu$ is assumed to hold. The ideas can clearly be accommodated to the dual model in Section 3.2, but the details are omitted. Interested readers are also referred to e.g. Dickson and Li (2013, Section 4.2) for the analysis of the distribution of the number of periods of negative surplus in a Sparre Andersen insurance risk model with $\operatorname{Erlang}(2)$ inter-arrival times.

Let $Q=\sup \left\{n \in\{0\} \cup \mathbb{N}: \tau_{U_{S}}^{-, n}<\infty\right\}$ be the total number of periods of negative surplus (assuming that the surplus process $\left\{U_{S}(t)\right\}_{t \geq 0}$ continues forever). Note that the case $Q=0$ (as $\left.\tau_{U_{S}}^{-, 0}=0\right)$ is simply the situation where ruin does not occur (i.e. $T_{U_{S}}=\infty$ ). In the present context, although a negative surplus leads to ruin according to the traditional definition, it may or may not result in Parisian ruin. Hence, we further define $Q_{P}=\sum_{n=1}^{Q} 1_{\left\{\tau_{U_{S}}^{+, n}-\tau_{U_{S}^{-, n}} \geq d\right\}}$ and $Q_{\bar{P}}=\sum_{n=1}^{Q} 1_{\left\{\tau_{U_{S}}^{+, n}-\tau_{U_{S}}^{-, n}<d\right\}}$ to be the numbers of periods of negative surplus that are of duration more than $d$ and less than $d$ respectively (such that $\left.Q=Q_{P}+Q_{\bar{P}}\right)$. We are interested in obtaining the joint probability mass function of the bivariate random vector $\left(Q_{P}, Q_{\bar{P}}\right)$, namely $\mathbb{P}^{u}\left\{N_{P}=m, N_{\bar{P}}=n\right\}$ for $m, n=0,1,2, \ldots$ First, the case $m=n=0$ is trivial because it corresponds to the event $\{Q=0\}$ and hence

$$
\mathbb{P}^{u}\left\{Q_{P}=0, Q_{\bar{P}}=0\right\}=1-\phi_{0} e^{-\mu\left(1-\phi_{0}\right) u},
$$

which is the traditional survival probability (see (3.2)). Concerning other values of $(m, n)$, the same arguments as in Section 3.1 are applicable. For $m=1,2, \ldots$, we have

$$
\mathbb{P}^{u}\left\{Q_{P}=m, Q_{\bar{P}}=0\right\}=\mathbb{P}^{u}\left\{B_{1}\right\}\left(\mathbb{P}^{*}\left\{C_{1}^{c} \cap B_{2}\right\}\right)^{m-1} \mathbb{P}^{*}\left\{C_{1}^{c} \cap B_{2}^{c}\right\} .
$$

The above equation allows for the following interpretation. In order for $\left\{U_{S}(t)\right\}_{t \geq 0}$ to have $m$ periods of negative surplus (where $m=1,2, \ldots$ ), all of which are of duration no less than $d$, the following should happen.

- The process $\left\{U_{S}(t)\right\}_{t \geq 0}$ should first drop below zero, and this happens with probability $\mathbb{P}^{u}\left\{B_{1}\right\}$.

- Recovery of the process to level zero should happen in no less than $d$ time units (i.e. event $C_{1}^{c}$ ), and then the process has to drop below zero again (i.e. event $B_{2}$ ) in order for the next period of negative surplus to kick in. This whole thing happens independently for a total of $m-1$ times, which explains the term $\left(\mathbb{P}^{*}\left\{C_{1}^{c} \cap B_{2}\right\}\right)^{m-1}$.

- After the final period of negative surplus, which is also of time length no less than $d$, the process drifts to infinity without ever dropping below zero. This constitutes the probability $\mathbb{P}^{*}\left\{C_{1}^{c} \cap B_{2}^{c}\right\}$.

While $\mathbb{P}^{u}\left\{B_{1}\right\}$ in (4.1) is the traditional ruin probability which is a special case of (3.2) with $\delta=0$, the other two probabilities are easily found to be

$$
\mathbb{P}^{*}\left\{C_{1}^{c} \cap B_{2}\right\}=\int_{0}^{\infty} \int_{0}^{y}\left(\int_{\frac{y}{c}+d}^{\infty} f_{Z_{D}}(t, y \mid 0) \mathrm{d} t\right)\left(\mu e^{-\mu x}\right)\left(\phi_{0} e^{-\mu\left(1-\phi_{0}\right)(y-x)}\right) \mathrm{d} x \mathrm{~d} y
$$




$$
\begin{aligned}
& +\int_{0}^{\infty} \int_{y}^{\infty}\left(\int_{\frac{y}{c}+d}^{\infty} f_{Z_{D}}(t, y \mid 0) \mathrm{d} t\right)\left(\mu e^{-\mu x}\right) \mathrm{d} x \mathrm{~d} y \\
= & \int_{0}^{\infty} e^{-\mu\left(1-\phi_{0}\right) y} \int_{\frac{y}{c}+d}^{\infty} f_{Z_{D}}(t, y \mid 0) \mathrm{d} t \mathrm{~d} y \\
\mathbb{P}^{*}\left\{C_{1}^{c} \cap B_{2}^{c}\right\}= & \int_{0}^{\infty} \int_{0}^{y}\left(\int_{\frac{y}{c}+d}^{\infty} f_{Z_{D}}(t, y \mid 0) \mathrm{d} t\right)\left(\mu e^{-\mu x}\right)\left(1-\phi_{0} e^{-\mu\left(1-\phi_{0}\right)(y-x)}\right) \mathrm{d} x \mathrm{~d} y \\
= & \int_{0}^{\infty}\left(1-e^{-\mu\left(1-\phi_{0}\right) y}\right) \int_{\frac{y}{c}+d}^{\infty} f_{Z_{D}}(t, y \mid 0) \mathrm{d} t \mathrm{~d} y .
\end{aligned}
$$

Next, similar to (4.1), for $n=1,2,3, \ldots$, we arrive at

$$
\mathbb{P}^{u}\left\{Q_{P}=0, Q_{\bar{P}}=n\right\}=\mathbb{P}^{u}\left\{B_{1}\right\}\left(\mathbb{P}^{*}\left\{C_{1} \cap B_{2}\right\}\right)^{n-1} \mathbb{P}^{*}\left\{C_{1} \cap B_{2}^{c}\right\},
$$

where $\mathbb{P}^{*}\left\{C_{1} \cap B_{2}\right\}$ is simply a special case of (3.3) with $\delta=0$, and $\mathbb{P}^{*}\left\{C_{1} \cap B_{2}^{c}\right\}$ can be evaluated as

$$
\begin{aligned}
\mathbb{P}^{*}\left\{C_{1} \cap B_{2}^{c}\right\} & =\int_{0}^{\infty} \int_{0}^{y}\left(\int_{\frac{y}{c}}^{\frac{y}{c}+d} f_{Z_{D}}(t, y \mid 0) \mathrm{d} t\right)\left(\mu e^{-\mu x}\right)\left(1-\phi_{0} e^{-\mu\left(1-\phi_{0}\right)(y-x)}\right) \mathrm{d} x \mathrm{~d} y \\
& =\int_{0}^{\infty}\left(1-e^{-\mu\left(1-\phi_{0}\right) y}\right) \int_{\frac{y}{c}}^{\frac{y}{c}+d} f_{Z_{D}}(t, y \mid 0) \mathrm{d} t \mathrm{~d} y .
\end{aligned}
$$

Finally, for $m, n=1,2, \ldots$, we have

$$
\begin{aligned}
\mathbb{P}^{u}\left\{Q_{P}=m, Q_{\bar{P}}=n\right\}= & \left(\begin{array}{c}
m+n-1 \\
m
\end{array}\right) \mathbb{P}^{u}\left\{B_{1}\right\}\left(\mathbb{P}^{*}\left\{C_{1} \cap B_{2}\right\}\right)^{n-1}\left(\mathbb{P}^{*}\left\{C_{1}^{c} \cap B_{2}\right\}\right)^{m} \mathbb{P}^{*}\left\{C_{1} \cap B_{2}^{c}\right\} \\
& +\left(\begin{array}{c}
m+n-1 \\
n
\end{array}\right) \mathbb{P}^{u}\left\{B_{1}\right\}\left(\mathbb{P}^{*}\left\{C_{1} \cap B_{2}\right\}\right)^{n}\left(\mathbb{P}^{*}\left\{C_{1}^{c} \cap B_{2}\right\}\right)^{m-1} \mathbb{P}^{*}\left\{C_{1}^{c} \cap B_{2}^{c}\right\},
\end{aligned}
$$

where all probabilities have already been determined. Note that the total number of periods of negative surplus is $m+n$. In the above expression, the first term is contributed by the case where the final period of negative surplus is of length less than $d$. In this case, the first $m+n-1$ periods of negative surplus should consist of $m$ periods of length no less than $d$ along with $n-1$ periods that are shorter than $d$, resulting in the combinatorial factor. The second term in (4.2) can be interpreted in the same manner.

\section{Random Parisian delay and occupation time in red}

It is instructive to note that the techniques used in previous sections are also applicable to the case where the Parisian delay $d$ is random instead. Similar to Section 4 , we only demonstrate the ideas through the Sparre Andersen insurance risk model with exponential claims. Specifically, when $\left\{U_{S}(t)\right\}_{t \geq 0}$ falls below zero for the $n$-th time, a random variable $d_{n}$ representing the $n$-th Parisian delay is generated. The delays are assumed to form an i.i.d. sequence independent of $\left\{U_{S}(t)\right\}_{t \geq 0}$. The definition of the Parisian ruin time (2.1) becomes $\tau_{U_{S}}^{\mathrm{r}}=\tau_{U_{S}}^{-, L^{\mathrm{r}}}+d_{L^{\mathrm{r}}}$, where $L^{\mathrm{r}}=\inf \left\{n \in \mathbb{N}: \tau_{U_{S}^{+}}^{+, n}-\tau_{U_{S}}^{-, n} \geq d_{n}\right\}$ (and it is understood that $\tau_{U_{S}}^{\mathrm{r}}=\infty$ if $\left.L^{\mathrm{r}}=\infty\right)$, and the Laplace transform of $L^{\mathrm{r}}$ is denoted by $\psi_{\delta, U_{S}}^{\mathrm{r}}(u)$. Here the superscript ' $r$ ' means 'random delay'. Then the arguments leading to (3.5) are still valid, and we only need to modify

the result by mixing the expectations in each excursion over $d$. In particular, if each delay is continuous with density $g(\cdot)$, then $\psi_{\delta, U_{S}}^{\mathrm{r}}(u)$ can be represented as

$$
\psi_{\delta, U_{S}}^{\mathrm{r}}(u)=\mathbb{E}^{u}\left[e^{-\delta \tau_{U_{S}}^{\mathrm{r}}} 1_{\left\{\tau_{U_{S}}^{\mathrm{r}}<\infty\right\}}\right]=\frac{\mathbb{E}^{u}\left[e^{-\delta L_{+, 1}} 1_{B_{1}}\right] \int_{0}^{\infty} \mathbb{E}^{*}\left[e^{-\delta d_{1}} 1_{C_{1}^{c}}\right] g(d) \mathrm{d} d}{1-\int_{0}^{\infty} \mathbb{E}^{*}\left[e^{-\delta\left(L_{-, 1}+L_{+, 2}\right)} 1_{C_{1} \cap B_{2}}\right] g(d) \mathrm{d} d},
$$


where the expectations involved are given by (3.2)-(3.4).

In the remainder of this section, it is assumed that each delay is exponentially distributed with mean $1 / \omega$. Thanks to the memoryless property of exponential delay, such a model is equivalent to

- the Omega model (e.g. Gerber et al. (2012)) in which bankruptcy is declared at rate $\omega$ whenever the surplus process $\left\{U_{S}(t)\right\}_{t \geq 0}$ is negative (and bankruptcy corresponds to Parisian ruin); and

- a model with randomized observations (e.g. Albrecher et al. (2013)) in which ruin is only checked at time points that are the arrival epochs of a Poisson process with rate $\omega$.

Therefore, the present model can be used to analyze occupation times as follows. Denoting an exponential random variable (independent of $\left\{U_{S}(t)\right\}_{t \geq 0}$ and the delays) with mean $1 / \delta$ by $\mathbf{e}_{\delta}$, it is known from e.g. Gerber et al. (2012, Section 4) or Li and Zhou (2013, Equation (4.2)) that

$$
\mathbb{E}^{u}\left[e^{-\omega \int_{0}^{\mathbf{e}_{\delta}} 1_{\left\{U_{S}(t)<0\right\}} \mathrm{d} t}\right]=1-\psi_{\delta, U_{S}}^{\mathrm{e}}(u),
$$

where $\int_{0}^{\mathbf{e}_{\delta}} 1_{\left\{U_{S}(t)<0\right\}} \mathrm{d} t$ counts (up to time $\mathbf{e}_{\delta}$ ) the amount of time when $\left\{U_{S}(t)\right\}_{t \geq 0}$ is negative, and $\psi_{\delta, U_{S}}^{\mathrm{e}}(u)$ is the Laplace transform of the Parisian ruin time (with 'e' meaning exponential delay). While the left-hand side of (5.2) represents the Laplace transform of the afore-mentioned occupation time (or time in red) with Laplace transform argument $\omega$, the quantity $\psi_{\delta, U_{S}}^{\mathrm{e}}(u)$ can be computed via (5.1) under $g(d)=\omega e^{-\omega d}$ for $d>0$. An important special case of (5.2) is obtainable by letting $\delta \rightarrow 0^{+}$so that

$$
\mathbb{E}^{u}\left[e^{-\omega \int_{0}^{\infty} 1_{\left\{U_{S}(t)<0\right\}} \mathrm{d} t}\right]=1-\psi_{0, U_{S}}^{\mathrm{e}}(u),
$$

i.e. the Laplace transform of the time in red over an infinite time horizon equals the Parisian survival probability.

Now, we shall explicitly evaluate the right-hand side of (5.1) when $g(d)=\omega e^{-\omega d}$. First, substitution of (3.4) followed by a change of order of integrations leads the integral in the numerator to

$$
\begin{aligned}
\int_{0}^{\infty} \mathbb{E}^{*}\left[e^{-\delta d} 1_{C_{1}^{c}}\right] \omega e^{-\omega d} \mathrm{~d} d & =\int_{0}^{\infty} \int_{\frac{y}{c}}^{\infty}\left(\int_{0}^{t-\frac{y}{c}} \omega e^{-(\omega+\delta) d} \mathrm{~d} d\right) f_{Z_{D}}(t, y \mid 0) \mathrm{d} t \mathrm{~d} y \\
& =\frac{\omega}{\omega+\delta} \int_{0}^{\infty} \int_{\frac{y}{c}}^{\infty} f_{Z_{D}}(t, y \mid 0) \mathrm{d} t \mathrm{~d} y-\frac{\omega}{\omega+\delta} \int_{0}^{\infty} \int_{\frac{y}{c}}^{\infty} e^{-(\omega+\delta)\left(t-\frac{y}{c}\right)} f_{Z_{D}}(t, y \mid 0) \mathrm{d} t \mathrm{~d} y
\end{aligned}
$$

Note that the first double integral above equals one under the loading condition $c \mathbb{E}\left[V_{1}\right]>1 / \mu$ of the insurance risk model. Further utilizing (2.8) and (2.9), we arrive at

$$
\begin{aligned}
\int_{0}^{\infty} \mathbb{E}^{*}\left[e^{-\delta d} 1_{C_{1}^{c}}\right] \omega e^{-\omega d} \mathrm{~d} d & =\frac{\omega}{\omega+\delta}-\frac{\omega}{\omega+\delta} \int_{0}^{\infty} e^{\frac{\omega+\delta}{c} y} h_{\omega+\delta, Z_{D}}(y \mid 0) \mathrm{d} y \\
& =\frac{\omega}{\omega+\delta}-\frac{\omega \mu}{\omega+\delta} \int_{0}^{\infty} \int_{\frac{y}{c}}^{\infty} e^{-\left(R_{\omega+\delta}+\frac{\omega+\delta}{c}\right)(c t-y)} k(t) \mathrm{d} t \mathrm{~d} y,
\end{aligned}
$$

where $R_{\omega+\delta}$ is defined by the Lundberg's equation (2.10) but with $\delta$ replaced by $\omega+\delta$. Straightforward calculation of the double integral results in

$$
\int_{0}^{\infty} \mathbb{E}^{*}\left[e^{-\delta d} 1_{C_{1}^{c}}\right] \omega e^{-\omega d} \mathrm{~d} d=\frac{\omega}{\omega+\delta}-\frac{\omega \mu}{\omega+\delta} \frac{1}{R_{\omega+\delta}+\frac{\omega+\delta}{c}}\left[1-\widetilde{k}\left(c R_{\omega+\delta}+\omega+\delta\right)\right]
$$


Because of the Lundberg's equation (2.10), the term inside the square bracket equals $R_{\omega+\delta} / \mu$, and therefore the above expression can be simplified to yield

$$
\int_{0}^{\infty} \mathbb{E}^{*}\left[e^{-\delta d} 1_{C_{1}^{c}}\right] \omega e^{-\omega d} \mathrm{~d} d=\frac{\omega}{c R_{\omega+\delta}+\omega+\delta} .
$$

Next, we omit the similar procedure and state that the integral in the denominator of (5.1) is found to be

$$
\int_{0}^{\infty} \mathbb{E}^{*}\left[e^{-\delta\left(L_{-, 1}+L_{+, 2}\right)} 1_{C_{1} \cap B_{2}}\right] \omega e^{-\omega d} \mathrm{~d} d=\frac{c\left(R_{\omega+\delta}-R_{\delta}\right)}{c\left(R_{\omega+\delta}-R_{\delta}\right)+\omega} .
$$

Finally, (3.2), (5.3) and (5.4) are substituted into (5.1). After some simplifications along with the use of $\phi_{\delta}=\left(\mu-R_{\delta}\right) / \mu$, we obtain

$$
\psi_{\delta, U_{S}}^{\mathrm{e}}(u)=\frac{c\left(R_{\omega+\delta}-R_{\delta}\right)+\omega}{c R_{\omega+\delta}+\omega+\delta} \frac{\mu-R_{\delta}}{\mu} e^{-R_{\delta} u}
$$

which is explicit in terms of model parameters and the Lundberg's roots $R_{\delta}$ and $R_{\omega+\delta}$.

Remark 2 If it is additionally assumed that the claims counting process $\{N(t)\}_{t \geq 0}$ is a Poisson process with rate $\lambda$ (so that $k(t)=\lambda e^{-\lambda t}$ for $t>0$ and $\widetilde{k}(s)=\lambda /(\lambda+s)$ ), then our model is equivalent to the one in Albrecher et al. (2013, Example 2.1). In this case, (5.5) reduces to

$$
\psi_{\delta, U_{S}}^{\mathrm{e}}(u)=\left(1-\frac{c R_{\delta}+\delta}{c R_{\omega+\delta}+\omega+\delta}\right) \frac{\mu-R_{\delta}}{\mu} e^{-R_{\delta} u}=\left(1-\frac{\frac{\lambda R_{\delta}}{\mu-R_{\delta}}}{\frac{\lambda R_{\omega+\delta}}{\mu-R_{\omega+\delta}}}\right) \frac{\mu-R_{\delta}}{\mu} e^{-R_{\delta} u}=\left(1-\frac{R_{\delta}}{R_{\omega+\delta}}\right) e^{-R_{\delta} u},
$$

where the Lundberg's equation (2.10) has been applied in the second equality. The above equation is consistent with Equation (2.18) in Albrecher et al. (2013) when $u \geq 0$.

Remark 3 Suppose that each delay is more generally distributed as a combination of exponentials with density $g(d)=\sum_{i=1}^{m} p_{i} \omega_{i} e^{-\omega_{i} d}$ for $y>0$, where $\sum_{i=1}^{m} p_{i}=1$ and $\omega_{i}$ 's are positive parameters. The quantity $\psi_{\delta, U_{S}}^{\mathrm{r}}(u)$ in (5.1) can still be readily calculated using the above results. Utilizing (5.3) and (5.4), one concludes that the two integrals in (5.1) are simply

$$
\begin{aligned}
\int_{0}^{\infty} \mathbb{E}^{*}\left[e^{-\delta d} 1_{C_{1}^{c}}\right] g(d) \mathrm{d} d & =\sum_{i=1}^{m} p_{i} \frac{\omega_{i}}{c R_{\omega_{i}+\delta}+\omega_{i}+\delta} . \\
\int_{0}^{\infty} \mathbb{E}^{*}\left[e^{-\delta\left(L_{-, 1}+L_{+, 2}\right)} 1_{C_{1} \cap B_{2}}\right] g(d) \mathrm{d} d & =\sum_{i=1}^{m} p_{i} \frac{c\left(R_{\omega_{i}+\delta}-R_{\delta}\right)}{c\left(R_{\omega_{i}+\delta}-R_{\delta}\right)+\omega_{i}} .
\end{aligned}
$$

\section{Example: Erlang(2) insurance risk model}

In this section, we consider the Erlang(2) insurance risk model with exponential claims and demonstrate how the Laplace transform of the Parisian ruin time $\psi_{\delta, U_{S}}(u)$ can be computed. The results in Section 3.1 are applicable by assuming that the interclaim times follow an Erlang(2) distribution with density $k(t)=\lambda^{2} t e^{-\lambda t}$ for $t>0$ and Laplace transform $\widetilde{k}(s)=[\lambda /(\lambda+s)]^{2}$. According to (3.5), we need to 
determine $\mathbb{E}^{u}\left[e^{-\delta L_{+, 1}} 1_{B_{1}}\right], \mathbb{E}^{*}\left[e^{-\delta\left(L_{-, 1}+L_{+, 2}\right)} 1_{C_{1} \cap B_{2}}\right]$ and $\mathbb{E}^{*}\left[e^{-\delta d} 1_{C_{1}^{c}}\right]$ explicitly. The first term is simply given by $(3.2)$, where $\phi_{\delta}$ ( such that $0<\phi_{\delta}<1$ ) can be solved from

$$
\phi_{\delta}=\left(\frac{\lambda}{\lambda+\delta+c \mu\left(1-\phi_{\delta}\right)}\right)^{2} .
$$

The evaluation of the remaining two expectations via (3.3) and (3.4) are more involved, as we need to apply the density $f_{Z_{D}}(t, y \mid 0)$ given by $(2.15)$. Because the $n$-fold convolution $k^{* n}(\cdot)$ corresponds to an $\operatorname{Erlang}(2 n)$ density, simplifications of $(2.15)$ yield

$$
\begin{aligned}
& f_{Z_{D}}(t, y \mid 0) \\
= & \mu e^{-\mu(c t-y)}\left[\lambda^{2} t e^{-\lambda t}+\sum_{n=1}^{\infty} \frac{\mu^{n}}{n !}(c t-y)^{n-1} \int_{\frac{y}{c}}^{t}(c z-y)\left(\frac{\lambda^{2 n}}{(2 n-1) !}(t-z)^{2 n-1} e^{-\lambda(t-z)}\right)\left(\lambda^{2} z e^{-\lambda z}\right) \mathrm{d} z\right] \\
= & \mu e^{-\mu(c t-y)} \lambda^{2} e^{-\lambda t}\left[t+\sum_{n=1}^{\infty} \frac{\left(\mu \lambda^{2} c^{-2}\right)^{n}}{(n+1) !(2 n+1) !}(c t-y)^{3 n}\left(t+\frac{n}{c} y\right)\right] \\
= & \mu e^{-\mu(c t-y)} \lambda^{2} e^{-\lambda t} \sum_{n=0}^{\infty} \frac{\left(\mu \lambda^{2} c^{-2}\right)^{n}}{(n+1) !(2 n+1) !}(c t-y)^{3 n}\left(t+\frac{n}{c} y\right) .
\end{aligned}
$$

Hence, (3.3) reduces to

$$
\begin{aligned}
& \mathbb{E}^{*}\left[e^{-\delta\left(L_{-, 1}+L_{+, 2}\right)} 1_{C_{1} \cap B_{2}}\right] \\
= & \sum_{n=0}^{\infty} \frac{\mu^{n+1} \lambda^{2 n+2} c^{-2 n}}{(n+1) !(2 n+1) !} \int_{0}^{\infty} e^{-\mu\left(1-\phi_{\delta}\right) y} \int_{\frac{y}{c}}^{\frac{y}{c}+d} e^{-(\lambda+\delta) t-\mu(c t-y)}(c t-y)^{3 n}\left(t+\frac{n}{c} y\right) \mathrm{d} t \mathrm{~d} y .
\end{aligned}
$$

The inner integral in the above equation can be expressed as

$$
\begin{aligned}
& \int_{\frac{y}{c}}^{\frac{y}{c}+d} e^{-(\lambda+\delta) t-\mu(c t-y)}(c t-y)^{3 n}\left(t+\frac{n}{c} y\right) \mathrm{d} t \\
= & \int_{0}^{c d} e^{-\frac{\lambda+\delta}{c}(x+y)-\mu x} x^{3 n}\left(\frac{x+y+n y}{c}\right) \frac{1}{c} \mathrm{~d} x \\
= & \frac{1}{c^{2}} e^{-\frac{\lambda+\delta}{c} y}\left(\int_{0}^{c d} e^{-\left(\frac{\lambda+\delta}{c}+\mu\right) x} x^{3 n+1} \mathrm{~d} x+y(n+1) \int_{0}^{c d} e^{-\left(\frac{\lambda+\delta}{c}+\mu\right) x} x^{3 n} \mathrm{~d} x\right) \\
= & \frac{1}{c^{2}} e^{-\frac{\lambda+\delta}{c} y}\left\{\left(\frac{c}{\lambda+\delta+c \mu}\right)^{3 n+2}[\Gamma(3 n+2)-\Gamma(3 n+2, d(\lambda+\delta+c \mu))]\right. \\
& \left.+y(n+1)\left(\frac{c}{\lambda+\delta+c \mu}\right)^{3 n+1}[\Gamma(3 n+1)-\Gamma(3 n+1, d(\lambda+\delta+c \mu))]\right\},
\end{aligned}
$$

where

$$
\Gamma(a)=\int_{0}^{\infty} t^{a-1} e^{-t} \mathrm{~d} t=(a-1) !, \quad a \in \mathbb{N},
$$

is the Gamma function and

$$
\Gamma(a, z)=\int_{z}^{\infty} t^{a-1} e^{-t} \mathrm{~d} t=(a-1) ! e^{-z} \sum_{k=0}^{a-1} \frac{z^{k}}{k !}, \quad a \in \mathbb{N},
$$


is the incomplete Gamma function. With (6.3), the double integral in (6.2) is given by

$$
\begin{aligned}
& \int_{0}^{\infty} e^{-\mu\left(1-\phi_{\delta}\right) y} \int_{\frac{y}{c}}^{\frac{y}{c}+d} e^{-(\lambda+\delta) t-\mu(c t-y)}(c t-y)^{3 n}\left(t+\frac{n}{c} y\right) \mathrm{d} t \mathrm{~d} y \\
= & \frac{c^{3 n}[\Gamma(3 n+2)-\Gamma(3 n+2, d(\lambda+\delta+c \mu))]}{(\lambda+\delta+c \mu)^{3 n+2}}\left(\mu\left(1-\phi_{\delta}\right)+\frac{\lambda+\delta}{c}\right)^{-1} \\
& +(n+1) \frac{c^{3 n-1}[\Gamma(3 n+1)-\Gamma(3 n+1, d(\lambda+\delta+c \mu))]}{(\lambda+\delta+c \mu)^{3 n+1}}\left(\mu\left(1-\phi_{\delta}\right)+\frac{\lambda+\delta}{c}\right)^{-2} \\
= & \frac{c^{3 n+1}[\Gamma(3 n+2)-\Gamma(3 n+2, d(\lambda+\delta+c \mu))]}{(\lambda+\delta+c \mu)^{3 n+2}\left[\lambda+\delta+c \mu\left(1-\phi_{\delta}\right)\right]}+(n+1) \frac{c^{3 n+1}[\Gamma(3 n+1)-\Gamma(3 n+1, d(\lambda+\delta+c \mu))]}{(\lambda+\delta+c \mu)^{3 n+1}\left[\lambda+\delta+c \mu\left(1-\phi_{\delta}\right)\right]^{2}},
\end{aligned}
$$

and therefore (6.2) becomes

$$
\begin{aligned}
\mathbb{E}^{*}\left[e^{-\delta\left(L_{-, 1}+L_{+, 2}\right)} 1_{C_{1} \cap B_{2}}\right]= & \sum_{n=0}^{\infty} \frac{\lambda^{2 n+2}(c \mu)^{n+1}[\Gamma(3 n+2)-\Gamma(3 n+2, d(\lambda+\delta+c \mu))]}{(n+1) !(2 n+1) !(\lambda+\delta+c \mu)^{3 n+2}\left[\lambda+\delta+c \mu\left(1-\phi_{\delta}\right)\right]} \\
& +\sum_{n=0}^{\infty} \frac{\lambda^{2 n+2}(c \mu)^{n+1}[\Gamma(3 n+1)-\Gamma(3 n+1, d(\lambda+\delta+c \mu))]}{n !(2 n+1) !(\lambda+\delta+c \mu)^{3 n+1}\left[\lambda+\delta+c \mu\left(1-\phi_{\delta}\right)\right]^{2}}
\end{aligned}
$$

Similarly, use of (6.1) and (6.3) leads (3.4) to

$$
\begin{aligned}
\mathbb{E}^{*}\left[e^{-\delta d} 1_{C_{1}^{c}}\right]= & e^{-\delta d}\left(1-\int_{0}^{\infty} \int_{\frac{y}{c}}^{\frac{y}{c}+d} f_{Z_{D}}(t, y \mid 0) \mathrm{d} t \mathrm{~d} y\right) \\
= & e^{-\delta d}\left(1-\sum_{n=0}^{\infty} \frac{\mu^{n+1} \lambda^{2 n+2} c^{-2 n}}{(n+1) !(2 n+1) !} \int_{0}^{\infty} \int_{\frac{y}{c}}^{\frac{y}{c}+d} e^{-\lambda t-\mu(c t-y)}(c t-y)^{3 n}\left(t+\frac{n}{c} y\right) \mathrm{d} t \mathrm{~d} y\right) \\
= & e^{-\delta d}\left(1-\sum_{n=0}^{\infty} \frac{\lambda^{2 n+1}(c \mu)^{n+1}[\Gamma(3 n+2)-\Gamma(3 n+2, d(\lambda+c \mu))]}{(n+1) !(2 n+1) !(\lambda+c \mu)^{3 n+2}}\right. \\
& \left.-\sum_{n=0}^{\infty} \frac{\lambda^{2 n}(c \mu)^{n+1}[\Gamma(3 n+1)-\Gamma(3 n+1, d(\lambda+c \mu))]}{n !(2 n+1) !(\lambda+c \mu)^{3 n+1}}\right) .
\end{aligned}
$$

To illustrate the computational tractability of our results, we consider a numerical example where each claim is exponential with mean 4 (i.e. $\mu=0.25$ ) and each interclaim time is Erlang(2) with mean 5 (i.e. $\lambda=0.4$ ). The incoming premium rate is assumed to be $c=1$, so that the positive security loading condition $2 c / \lambda>1 / \mu$ is satisfied. The Laplace transform of the Parisian ruin time $\psi_{\delta, U_{S}}(u)$ is given by formula (3.5) with the components computed via (3.2), (6.4) and (6.5). Using Mathematica, we plot $\psi_{\delta, U_{S}}(u)$ against the initial surplus $u$ for $\delta=0,0.02,0.04$ in Figures 5-7 respectively. Note that $\psi_{\delta, U_{S}}(u)$ is the Parisian ruin probability when $\delta=0$. Each of Figures 5-7 shows five curves for the values $d=0,1,2,3,4$, where $d=0$ corresponds to the traditional definition of ruin. From Figures $5-7$, it is clear that $\psi_{\delta, U_{S}}(u)$ is decreasing in $u$, as an increase in the initial surplus level keeps the surplus process $\left\{U_{S}(t)\right\}_{t \geq 0}$ further away from (Parisian) ruin. Within each figure, we observe that $\psi_{\delta, U_{S}}(u)$ decreases in $d$ as well. Intuitively, as $d$ increases, the insurer has a longer buffer period $d$ to recover from negative surplus when the surplus drops below zero, thereby lowering $\psi_{\delta, U_{S}}(u)$. Finally, comparison across Figures 5-7 reveals that $\psi_{\delta, U_{S}}(u)$ is also decreasing in $\delta$, which is clear from the definition (2.2). 


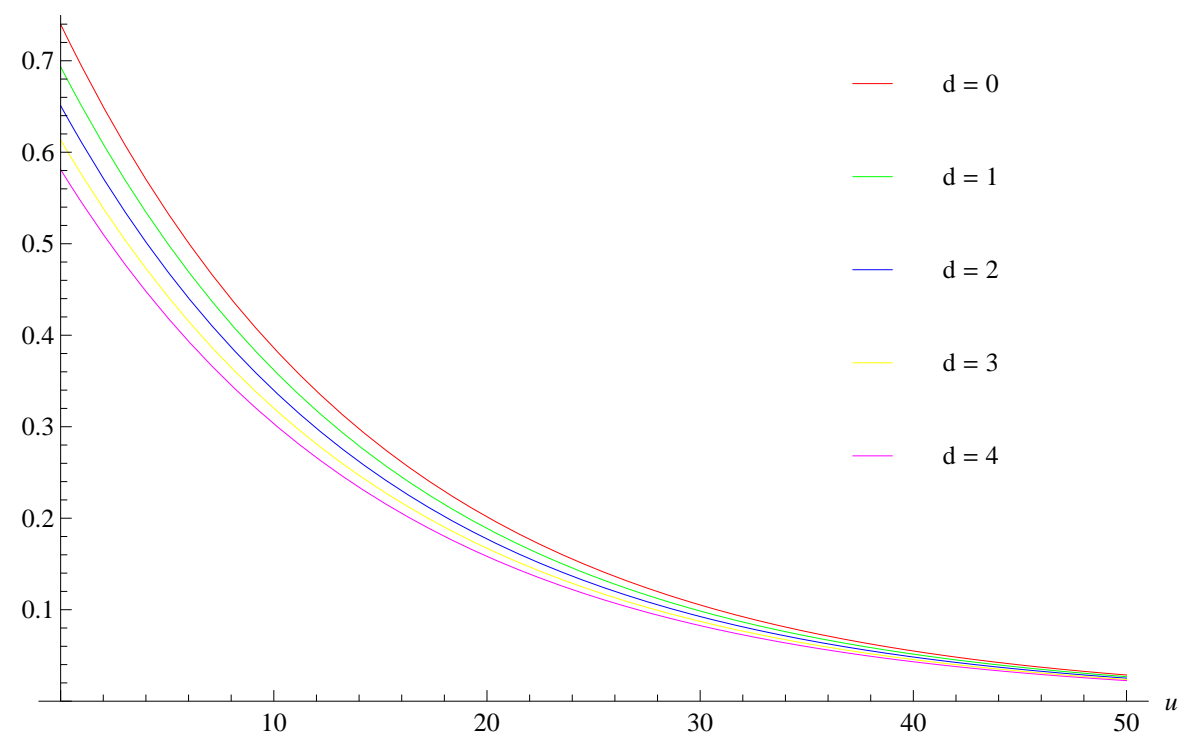

Figure 5: Parisian ruin probability

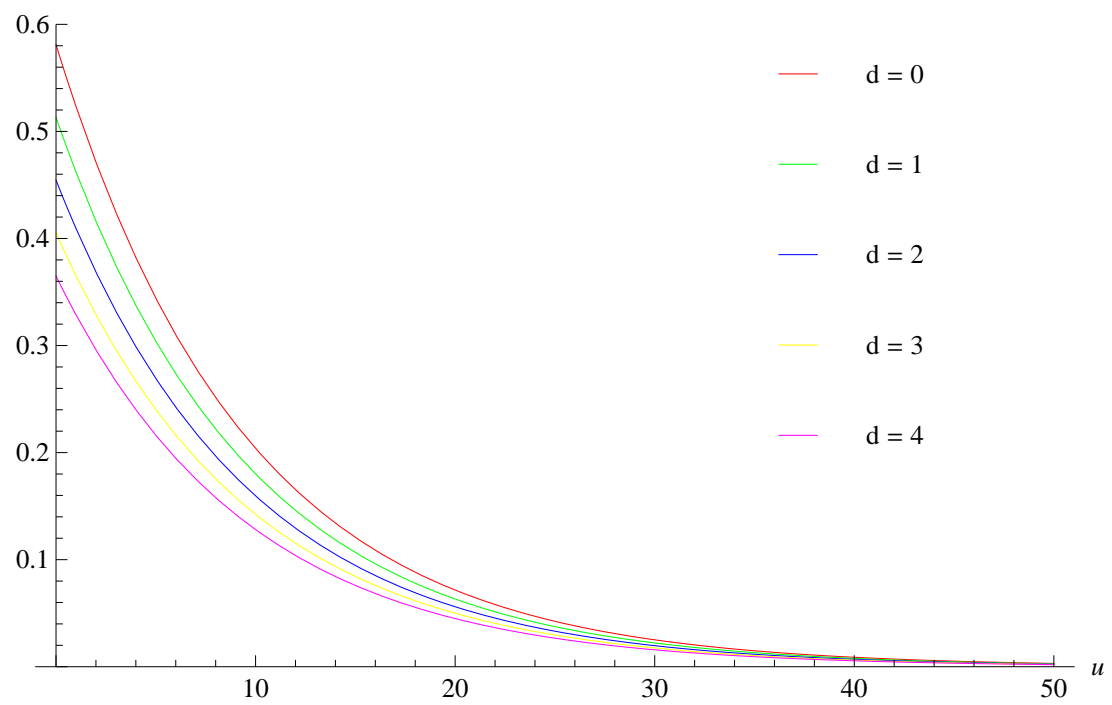

Figure 6: Laplace transform of Parisian ruin time under $\delta=0.02$

\section{Concluding remarks and open problems}

In contrast to the compound Poisson or more generally Lévy risk models in the literature, this paper analyzes the Parisian ruin problem in the (dual) renewal risk process with general inter-arrival times and exponential jumps. New results concerning the Laplace transform of the Parisian ruin time are derived under deterministic delays and then extended to random delays. Related formulas for the number of periods of negative surplus and the occupation time in red are given as well.

There are several directions for future research in the renewal model. First, one may try to incorporate 


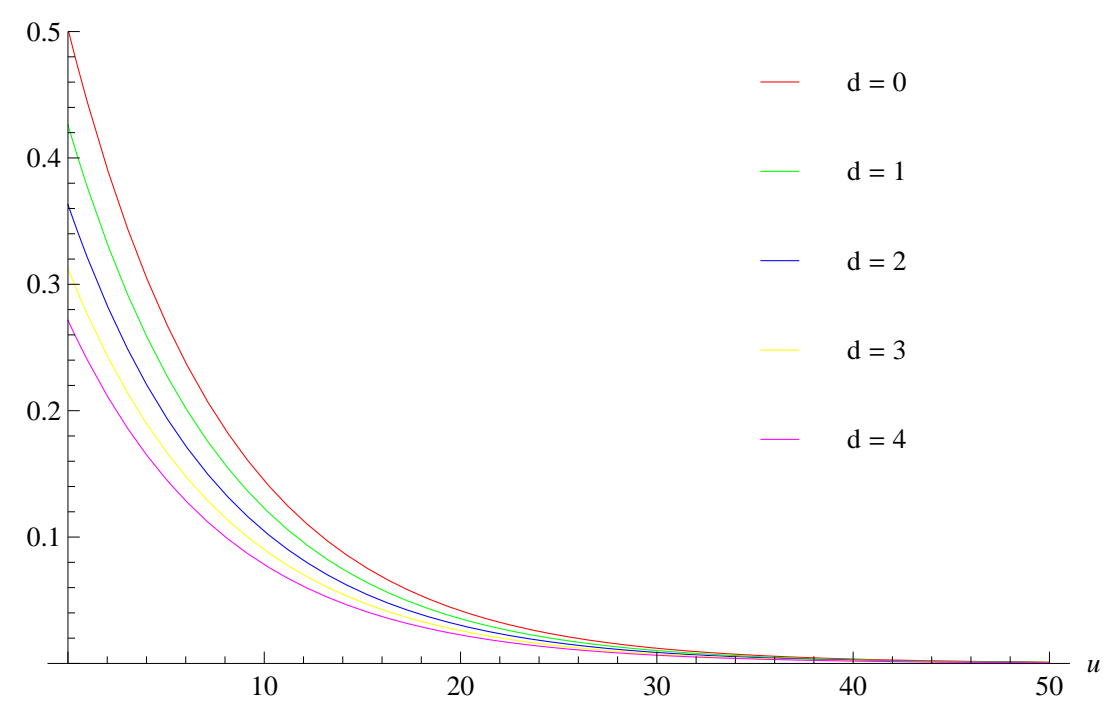

Figure 7: Laplace transform of Parisian ruin time under $\delta=0.04$

a lower default barrier $-a<0$ in the spirit of Czarna (2014). In such a case, in addition to the possibility of Parisian ruin, the surplus process is declared ruin immediately if it falls below $-a$. However, the analysis of the modified ruin time shall involve a two-sided exit problem for a renewal risk process where exact solutions are not easy to obtain. Second, it will also be interesting to study the distribution of the amount of shortfall at the Parisian ruin time. For the insurance model in Section 3.1, the quantity $\mathbb{P}\left\{Z_{D}(d) \in \mathrm{d} y, T_{Z_{D}}>d \mid Z_{D}\left(0^{-}\right)=0\right\}$ will be required. If the delay $d$ is exponential instead, then we need the Laplace transform (with respect to $d$ ) of the afore-mentioned quantity, which can be regarded as a potential measure for the process $\left\{Z_{D}(t)\right\}_{t \geq 0}$ defined in Section 2.2. We leave these as open research problems.

\section{Acknowledgements}

The authors would like to thank the anonymous referee for helpful comments and suggestions which improved an earlier version of the paper. Eric Cheung acknowledges the support from the Research Grants Council of the Hong Kong Special Administrative Region (Project Number: HKU 701212P). This research is also partially supported by the CAE 2013 research grant from the Society of Actuaries. Any opinions, finding, and conclusions or recommendations expressed in this material are those of the authors and do not necessarily reflect the views of the SOA.

\section{References}

[1] Albrecher, H., Badescu, A.L. and Landriault, D. 2008. On the dual risk model with tax payments. Insurance: Mathematics and Economics 42(3): 1086-1094.

[2] Albrecher, H., Cheung, E.C.K. and Thonhauser, S. 2011a. Randomized observation periods for the compound Poisson risk model: Dividends. ASTIN Bulletin 41(2): 645-672.

[3] Albrecher, H., Cheung, E.C.K. And Thonhauser, S. 2013. Randomized observation periods for the compound Poisson risk model: The discounted penalty function. Scandinavian Actuarial Journal 2013(6): 424-452. 
[4] Albrecher, H., Gerber, H.U. and Shiu, E.S.W. 2011b. The optimal dividend barrier in the Gamma-Omega model. European Actuarial Journal 1(1): 43-55.

[5] Albrecher, H. And Ivanovs, J. 2013. A risk model with an observer in a Markov environment. Risks 1(3): 148-161.

[6] Albrecher, H., Ivanovs, J. And Zhou, X. 2014. Exit identities for Lévy processes observed at Poisson arrival times. Bernoulli. To appear.

[7] Albrecher, H. and Lautscham, V. 2013. From ruin to bankruptcy for compound Poisson surplus processes. ASTIN Bulletin 43(2): 213-243.

[8] Albrecher, H. and Teugels, J.L. 2006. Exponential behavior in the presence of dependence in risk theory. Journal of Applied Probability 43(1): 257-273.

[9] Asmussen, S. And Albrecher, H. 2010. Ruin Probabilities. Second Edition. New Jersey: World Scientific.

[10] Avanzi, B., Gerber, H.U. And Shiu, E.S.W. 2007. Optimal dividends in the dual model. Insurance: Mathematics and Economics 41(1): 111-123.

[11] Borovkov, K.A. And Dickson, D.C.M. 2008. On the ruin time distribution for a Sparre Andersen process with exponential claim sizes. Insurance: Mathematics and Economics 42(3): 1104-1108.

[12] CAI, J. 2007. On the time value of absolute ruin with debit interest. Advances in Applied Probability $39(2): 343-359$.

[13] Cai, J., Feng, R. and Willmot, G.E. 2009. On the total discounted operating costs up to default and its applications. Advances in Applied Probability 41(2): 495-522.

[14] Chesney, M., Jeanblanc-Picqué, M. and Yor, M. 1997. Brownian excursions and Parisian barrier options. Advances in Applied Probability 29(1): 165-184.

[15] Cheung, E.C.K. 2011. A generalized penalty function in Sparre Andersen risk models with surplusdependent premium. Insurance: Mathematics and Economics 48(3): 384-397.

[16] Cheung, E.C.K. 2012. A unifying approach to the analysis of business with random gains. Scandinavian Actuarial Journal 2012(3): 153-182.

[17] Cheung, E.C.K. 2013. Moments of discounted aggregate claim costs until ruin in a Sparre Andersen risk model with general interclaim times. Insurance: Mathematics and Economics 53(2): 343-354.

[18] Cheung, E.C.K., Landriault, D., Willmot, G.E. and Woo, J.-K. 2010. Structural properties of Gerber-Shiu functions in dependent Sparre Andersen models. Insurance: Mathematics and Economics 46(1): 117-126.

[19] Cramér, H. 1955. Collective Risk Theory. Jubilee volume of Forsakringsbolaget Skandia, Stockholm.

[20] Czarna, I. 2014. Parisian ruin probability with a lower ultimate bankrupt barrier. Scandinavian Actuarial Journal. In press.

[21] Czarna, I. and Palmowski, Z. 2011. Ruin probability with Parisian delay for a spectrally negative Lévy risk process. Journal of Applied Probability 48(4): 984-1002. 
[22] Czarna, I., Palmowski, Z. And ŚwiA̧tek, P. 2014. Binomial discrete time ruin probability with Parisian delay. Preprint available at http://arxiv.org/pdf/1403.7761v1.pdf.

[23] Dassios, A. And Embrechts, P. 1989. Martingales and insurance risk. Communications in Statistics - Stochastic Models 5(2): 181-217.

[24] Dassios, A. And Wu, S. 2008a. Parisian ruin with exponential claims. Preprint available at http://stats.lse.ac.uk/angelos/docs/exponentialjump.pdf.

[25] Dassios, A. And Wu, S. 2008b. Ruin probabilities of the Parisian type for small claims. Preprint available at http://stats.lse.ac.uk/angelos/docs/paper5a.pdf.

[26] Dickson, D.C.M. And Hipp, C. 2001. On the time to ruin for Erlang(2) risk processes. Insurance: Mathematics and Economics 29(3): 333-344.

[27] Dickson, D.C.M., Hughes, B.D. And Zhang, L. 2005. The density of the time to ruin for a Sparre Andersen process with Erlang arrivals and exponential claims. Scandinavian Actuarial Journal 2005(5): 358-376.

[28] Dickson, D.C.M. And Li, S. 2010. Finite time ruin problems for the Erlang(2) risk model. Insurance: Mathematics and Economics 46(1): 12-18.

[29] Dickson, D.C.M. And Li, S. 2013. The distribution of the time to reach a given level and the duration of negative surplus in the Erlang(2) risk model. Insurance: Mathematics and Economics 52(3): 490-497.

[30] Dickson, D.C.M. And Willmot, G.E. 2005. The density of the time to ruin in the classical Poisson risk model. ASTIN Bulletin 35(1): 45-60.

[31] Drekic, S. And Willmot, G.E. On the density and moments of the time of ruin with exponential claims. ASTIN Bulletin 33(1): 11-21.

[32] Egidio dos Reis, A.D. 1993. How long is the surplus below zero? Insurance: Mathematics and Economics 12(1): 23-28.

[33] Gerber, H.U. 1990. When does the surplus reach a given target? Insurance: Mathematics and Economics 9(2): 115-119.

[34] Gerber, H.U. And Shiu, E.S.W. 1998. On the time value of ruin. North American Actuarial Journal 2(1): 48-78.

[35] Gerber, H.U. And Shiu, E.S.W. 2005. The time value of ruin in a Sparre Andersen model. North American Actuarial Journal 9(2): 49-69.

[36] Gerber, H.U., Shiu, E.S.W. And Yang, H. 2012. The Omega model: From bankruptcy to occupation times in the red. European Actuarial Journal 2(2): 259-272.

[37] Gerber, H.U. And H. Yang. 2007. Absolute ruin probabilities in a jump diffusion risk model with investment. North American Actuarial Journal 11(3): 159-169.

[38] Landriault, D., Renaud, J.-F. And Zhou, X. 2014. An insurance risk model with Parisian implementation delays. Methodology and Computing in Applied Probability 16(3): 583-607. 
[39] Li, B. And Zhou, X. 2013. The joint Laplace transforms for diffusion occupation times. Advances in Applied Probability 45(4): 1049-1067.

[40] Li, S. AND Garrido, J. 2004. On ruin for the $\operatorname{Erlang}(n)$ risk process. Insurance: Mathematics and Economics 34(3): 391-408.

[41] Loeffen, R., Czarna, I. And Palmowski, Z. 2013. Parisian ruin probability for spectrally negative Lévy processes. Bernoulli 19(2): 599-609.

[42] Mazza, C. And Rullière, D. 2004. A link between wave governed random motions and ruin processes. Insurance: Mathematics and Economics 35(2): 205-222.

[43] Prabhu N.U. 1998. Stochastic Storage Processes: Queues, Insurance Risk, Dams, and Data Communication. New York: Springer.

[44] Schröder, M. 2003. Brownian excursions and Parisian barrier options: A note. Journal of Applied Probability 40(4): 855-864.

[45] Seal, H.L. 1969. Stochastic Theory of a Risk Business. New York: Wiley.

[46] Sparre Andersen, E. 1957. On the collective theory of risk in the case of contagion between claims. In: Proceedings of the Transactions of the XVth International Congress on Actuaries vol. II, New York, pp. 219-229.

[47] TakÁcs, H. 1967. Combinatorial Methods in the Theory of Stochastic Processes. New York: Wiley.

[48] Willmot, G.E. 2007. On the discounted penalty function in the renewal risk model with general interclaim times. Insurance: Mathematics and Economics 41(1): 17-31.

[49] Willmot, G.E. And Woo, J.-K. 2010. Surplus analysis for a class of Coxian interclaim time distributions with applications to mixed Erlang claim amounts. Insurance: Mathematics and Economics 46(1): 32-41.

[50] Wong, J.T.Y. 2014. On some Parisian problems in ruin theory. MPhil Thesis, University of Hong Kong. 